\title{
Reconstruction of effective connectivity in the case of asymmetric phase distributions
}

\author{
Azamat Yeldesbay, ${ }^{\mathrm{a}, \mathrm{b}}{ }^{*}$, Gereon R. Fink ${ }^{\mathrm{b}, \mathrm{c}}$, and Silvia Daun ${ }^{\mathrm{a}, \mathrm{b},{ }^{* *}}$ \\ ${ }^{a}$ University of Cologne, Institute of Zoology, Heisenberg Research Group of \\ Computational Neuroscience - Modeling Neural Network Function \\ Zülpicher Str. 47b, 50674 Cologne, Germany \\ ${ }^{\mathrm{b}}$ Research Centre Jülich, Institute of Neuroscience and Medicine (INM-3) \\ Cognitive Neuroscience, 52425 Jülich, Germany \\ ${ }^{\mathrm{c}}$ University of Cologne, Department of Neurology \\ Medical Faculty and University Hospital Cologne \\ Kerpener Str. 62, 50937 Cologne, Germany \\ *Corresponding author: azamat.yeldesbay@uni-koeln.de (A.Yeldesbay) \\ ** Principal corresponding author: s.daun@fz-juelich.de (S.Daun)
}

Published in

Journal of Neuroscience Methods in February 2019, Volume 317, pp 94-107, DOI: https://doi.org/10.1016/j.jneumeth.2019.02.009

\begin{abstract}
Background:The interaction of different brain regions is supported by transient synchronization between neural oscillations at different frequencies. Different measures based on synchronization theory are used to assess the strength of the interactions from experimental data. One method of estimating the effective connectivity between brain regions, within the framework of the theory of weakly coupled phase oscillators, was implemented in Dynamic Causal Modelling (DCM) for phase coupling (Penny et al., 2009). However, the results of such an approach strongly depend on the observables used to reconstruct the equations (Kralemann et al., 2008). In particular, an asymmetric distribution of the observables could result in a false estimation of the effective connectivity between the network nodes.

New method:In this work we built a new modelling part into DCM for phase coupling, and extended it with a distortion function that accommodates departures from purely sinusoidal oscillations.

Results:By analysing numerically generated data sets with an asymmetric phase distribution, we demonstrated that the extended DCM for phase coupling with the additional modelling component, correctly estimates the coupling functions.

Comparison with existing methods: The new method allows for different intrinsic frequencies among coupled neuronal populations and provides results that do not depend on the distribution of the observables.

Conclusions:The proposed method can be used to analyse effective connectivity between brain regions within and between different frequency bands, to characterize m:n phase coupling, and to unravel underlying mechanisms of the transient synchronization.
\end{abstract}


Keywords: Dynamic Causal Modelling, Phase oscillators, n:m synchronization

\section{Introduction}

Complex behavior, e.g., a motor task, requires coordinated neural activity at different levels of the brain. The interaction of an ensemble of neurons within a specific brain region is reflected in measurements of electrophysiological experiments by oscillatory activity. Data suggest that the interaction between brain regions causes synchronization of these emerging oscillatory activities (Buzsáki et al., 2004; Schnitzler and Gross, 2005; Buzsáki, 2006). This synchronization has transient dynamics and occurs from small distances within a brain region up to long-ranges between distant brain regions (Varela et al., 2001; van Ede et al., 2018). The magnitude of this synchronization can quantify the interactions between brain regions. There are many ways of analysing the connectivity of a network of interconnected brain regions using bivariate analysis of the phases of the oscillatory signals (Greenblatt et al., 2012), such as the phase locking value (PLV) (Lachaux et al., 1999), phase transient entropy (Lobier et al., 2014), phase-amplitude coupling (Tort et al., 2010), and cross-frequency coupling (Hyafil et al., 2015). The measures used in these studies are based on the theory of synchronization and the theory of weakly coupled oscillators (Hoppensteadt and Izhikevich, 1997; Pikovsky et al., 2001).

Another approach measuring connectivity based on the reconstruction of the dynamics of phase interactions from experimental data was suggested first by Rosenblum and Pikovsky (2001). In this method the values of the phases of experimentally measured signals and their derivatives are used to reconstruct the effective phase connectivity and phase dynamics. As a result, one obtains not only the structure of a network and the directionality of its edges, but also an analytic form of the interaction function between the nodes. A similar concept was realized in several recent studies to reconstruct the phase dynamic equations using Bayesian inference (Stankovski et al., 2012; Duggento et al., 2012; Stankovski et al., 2015).

Penny et al. (2009) presented a new variant of Dynamic Causal Modelling (DCM) for the analysis of phase-coupled data. Dynamic causal modelling (DCM) is a Bayesian model selection and inversion framework for identifying the structure and directed connectivity among brain regions from measured time series (Friston et al., 2003). This framework uses variational Bayes to find the functional architecture, the implicit directed connectivity and, in some instances, the hidden neuronal states that best explain the data. This rests upon the inversion of a generative model using data obtained by a wide range of measurement techniques, such as functional magnetic resonance imaging (fMRI), electroencephalography (EEG), magnetoencephalography (MEG), and local field potentials (LFPs) (Stephan et al., 2010; Daunizeau et al., 2011).

Most of the research on phase analysis relies on the direct association of the phases of the signals with the phases used in the theoretical description of weakly coupled oscillators. This is a plausible assumption. However, Kralemann et al. $(2007,2008)$ showed that the phases of the signals measured in experiments are not uniquely defined and an asymmetric distribution of the measured phases (e.g. non-sine form of the signals) can lead to systematic errors in the 
reconstruction procedure of the coupling. Moreover, the impact of this issue has been explored in recent studies, where it was demonstrated that a non-sine form of the signal can lead to spurious connections (Belluscio et al., 2012; van Driel et al., 2015; Scheffer-Teixeira and Tort, 2016; Lozano-Soldevilla et al., 2016).

Kralemann et al. (2008) suggested a solution for this problem by introducing a transformation from an arbitrary measured phase to an uniquely defined phase variable. Moreover, they could show that using this unique phase variable an invariant description of the phase dynamics can be obtained. In subsequent studies the authors developed a toolbox ${ }^{1}$ to analyse the dynamics of a network of coupled phase oscillators (Kralemann et al., 2011) and extended the method from pairwise synchronization cases to triplet synchronization cases, which take into account the mutual phase interactions of three regions (Kralemann et al., 2013, 2014).

Since DCM for phase-coupled data (Penny et al., 2009) is not designed to reconstruct network architectures when the measured phases have asymmetric distributions, we, in this work, extended DCM for phase coupling by implementing a transformation to unique phase variables. Moreover, the extension is adapted to measure the interaction function within and between different frequencies.

The paper is organized as follows. We first briefly introduce the theoretical framework of the synchronization of weakly coupled phase oscillators and explain the procedure of the reconstruction of the phase dynamics from experimental data. In the next section we explain the difference of the dynamics of a uniquely defined phase variable and of a phase measured in an experiment. Further we give a brief description of DCM, its parts, and explain the details of the version of the DCM for phase coupling (Penny et al., 2009). In Section 2.6 we introduce our model and the new extension to DCM for phase coupling and discuss several technical and theoretical aspects of our approach. Finally, we test the new extension of DCM on synthetic data using a model of coupled phase oscillators and the neural mass model.

\section{Methods}

\subsection{Transient synchronization between brain regions}

Let us assume we are measuring the activity of two distant brain regions during the performance of some action (e.g. a motor task) as shown in Fig. 1(a)(1). Consider that these measured activities have oscillations as the result of simultaneous action of many neurons in both regions (2). The regions are transiently synchronous if after filtering around a given frequency band the oscillatory time courses have a similar form during a short period of time (3). One can estimate the magnitude of synchrony when the time courses are separated into amplitude and phase. The phase of the signals can be calculated by measuring the angle $\theta$ after Hilbert or wavelet transformation (4). At full synchronization the difference between the phases is constant or equal to zero. However, during a period of transient synchronization the interaction time is not long enough to set the phases of the signals to a constant difference. The dynamics of the

\footnotetext{
${ }^{1}$ The toolbox "Data Analysis with Models Of Coupled Oscillators (DAMOCO)" can be found here http://www.stat.physik.uni-potsdam.de/ mros/damoco.html
} 
phases during these transient synchronizations can be described by the model of weakly coupled oscillators, which we discuss in the next sections.
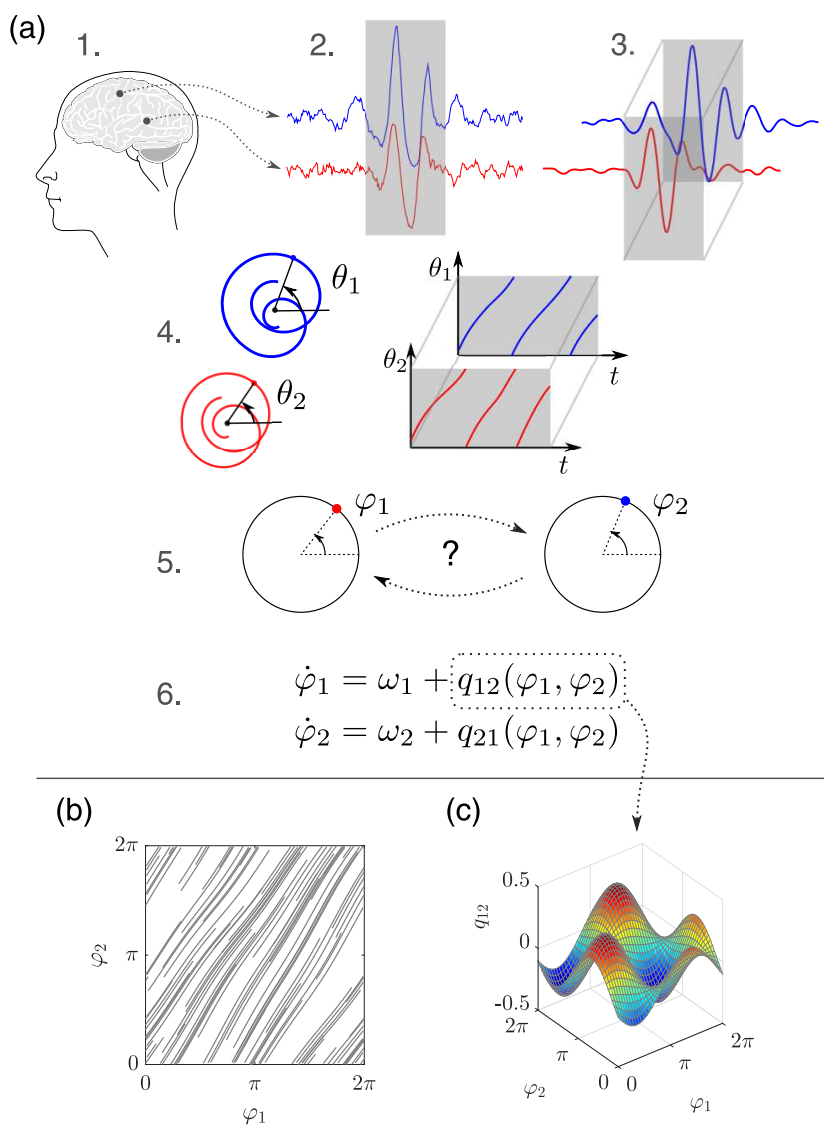

Figure 1: Measuring synchronization. (a) Scheme of the analysis of the connectivity between distant brain regions using the framework of weakly coupled oscillators. See text for details. (b) The trajectories of the trials on the $\left(\varphi_{1}, \varphi_{2}\right)$ surface (torus). (c) The coupling function $q_{12}\left(\varphi_{1}, \varphi_{2}\right)$ represented as a surface.

\subsection{Weakly coupled oscillators}

The theory of synchronization considers a model of weakly coupled phase oscillators (Kuramoto, 1984; Pikovsky et al., 2001). The theory shows that the dynamics of a self-sustained oscillatory system can be reduced to the ones of phase oscillators. Moreover, the theory suggests that the phase dynamics can capture the interaction between coupled oscillatory systems, since the phase, in contrast to the amplitude, can be adjusted through a very weak interaction. In this work we do not perform a phase reduction and the derivation of the equation for weakly coupled oscillators. However, we briefly introduce the main concepts necessary for this work.

If we consider $N$ uncoupled phase oscillators, then every oscillator would 
just rotate with its natural frequency $\omega$

$$
\dot{\varphi}_{i}=\omega_{i}, i=1 \ldots N,
$$

where $\varphi_{i}$ is the phase of the oscillator $i$. In the presence of coupling between the oscillators the equation reads

$$
\dot{\varphi}_{i}=\omega_{i}+F_{i}\left(\varphi_{1}, \ldots, \varphi_{N}\right)
$$

where the coupling function $F_{i}$, in general, depends on the phases of all oscillators. We consider here a simpler case with only pairwise coupling between the oscillators

$$
\dot{\varphi}_{i}=\omega_{i}+\sum_{j=1, j \neq i}^{N} q_{i j}\left(\varphi_{i}, \varphi_{j}\right) .
$$

The function $q_{i j}\left(\varphi_{i}, \varphi_{j}\right)$ is called the coupling function or the phase interaction function between the region $i$ and $j$. The Fourier series of the periodic coupling function $q_{i j}\left(\varphi_{i}, \varphi_{j}\right)$ has no constant term and no term that depends only on $\varphi_{i}$ - the phase of the oscillator the coupling function acts on.

\subsection{Reconstruction of the effective phase connectivity}

After reducing the oscillatory systems to weakly coupled phase oscillators, the interaction between brain regions is equivalent to the effective connectivity between phase oscillators (Fig. 1(a)(5)), which can be completely described by the coupling functions $q_{i j}$ (Stankovski et al., 2017). The procedure of the reconstruction of the effective phase connectivity is based on finding the coupling functions $q_{i j}$ for every pair of oscillators.

If we have the time series of phases for all oscillators, then, by calculating the derivative of the phases, we can find the right hand side of Eq. (3), i.e. the coupling functions (Rosenblum and Pikovsky, 2001). This can be explained by the following example for two phase oscillators (Fig. 1(a)(6)). The values of the phases from all trials can be presented on the surface of the plane (torus) $\left(\varphi_{1}, \varphi_{2}\right)$ as shown in Fig. 1(b). The procedure of reconstruction of the coupling function is then equivalent to finding the surface $q_{i j}\left(\varphi_{i}, \varphi_{j}\right)$ by calculating derivatives of the phase for every data point and subtracting the mean frequencies $\omega_{1}$ and $\omega_{2}$ (Fig. 1(c)). However, for a successful reconstruction of the coupling function the data points should fill the surface of the plane (torus) $\left(\varphi_{i}, \varphi_{j}\right)$ (Fig. 1(b)). This is not the case in full synchronization, when the trajectories of the different trials merge to a line (an attractor). Therefore, the method of reconstruction of the coupling functions is applicable only for a weak coupling between oscillators and/or for a transient synchronization. In the latter case the shortly active coupling is not enough to collapse all the trajectories to the attractor.

\subsection{Observable phase vs theoretical phase}

It was shown by Kralemann et al. $(2007,2008)$ that the phases measured in experiments obey the theoretical expression Eq. (3) only in exceptional cases and a special technique is needed to reconstruct the phase dynamics from the data. Following Kralemann et al. (2008) we briefly introduce the main idea of this technique. 
The expression Eq. (3) implies that without any coupling $\left(q_{i j}=0\right)$ the phase oscillator $\varphi_{i}$ rotates with constant frequency $\omega_{i}$. If we present the phase oscillator as a limit cycle, then the case without coupling corresponds to an oscillator with a circular limit cycle centered at the origin (Fig. 2(a)) and with a time course of one variable (e.g. $y$ ) in the form of a sine-wave function (Fig. 2(b)). We specify this phase as the theoretical or true phase. In the absence of coupling the theoretical phase grows linearly with a constant speed $\omega=2 \pi / T$, where $T$ is the period (Fig. 2(c)). Any non-linearity in growth is induced only by coupling.

In an experiment one measures a scalar observable that is a projection of the dynamics of a high dimensional system. For example, the dynamics of a twodimensional system on a limit cycle as presented in Fig. 2(d) could be measured in experiments as a regular oscillation of a variable $y$ as shown in Fig. 2 (e). In practice, the procedure is reversed: a corresponding two-dimensional limit cycle (Fig. 2 (d)) is reconstructed from the time series of the scalar observable (Fig. 2 (e)) using a phase estimation method, e.g. the Hilbert transformation (Pikovsky et al., 2001). The "phases" of the analysed system can be extracted by measuring the angle $\theta$ in the limit cycle (Fig. 2 (d)). We refer to this phase as an observable phase (also called proto-phase by Kralemann et al. $(2007,2008)$ ). The observable phase $\theta$ grows monotonically with the same period as the time series it is extracted from (Fig. 2 (f)). However, since the reconstructed limit cycle is generally not circular as in Fig. 2 (a) and depends on the method used to reconstruct it, the observable phase $\theta$ grows non-linearly even without coupling (!). Therefore, this non-linearity can cause spurious couplings in the reconstruction procedure of the coupling functions. Moreover, the reconstructed limit cycle and, respectively, the observable phase is not unique. They depend on the scalar observable and on the methods of limit cycle reconstruction and phase estimation, e.g. the Hilbert transformation, the Wavelet transformation, the Poincare section method (Kralemann et al., 2008).

The non-linear growth of the observable phase plays a crucial role in the reconstruction of the coupling function. To demonstrate this, let us consider two coupled oscillators with phases $\varphi_{1}$ and $\varphi_{2}$ that obey the equation of the form Eq. (3) where the coupling functions are $q_{i j}\left(\varphi_{1}, \varphi_{2}\right)=a_{i} \sin \left(\varphi_{i}-\varphi_{j}\right)$. If we numerically integrate Eq. (3) with different initial conditions we obtain the time series $\varphi_{1}(t)$ and $\varphi_{2}(t)$. As was discussed above, by calculating the derivatives of these time series we can obtain the right hand side of Eq. (3), i.e. the coupling functions in the form of surfaces shown in Fig. $2(\mathrm{~g})$ and $(\mathrm{j})$. Now let us assume that we are measuring not $\varphi_{1}(t)$ and $\varphi_{2}(t)$, but the observable phases $\theta_{1}$ and $\theta_{2}$, which have non-linear relations to $\varphi_{1}$ and $\varphi_{2}$ as shown in Fig. 2 (h) and (k). Using these relations we can find the time series of the observable phases $\theta_{1}(t)$ and $\theta_{2}(t)$. If we reconstruct the coupling function from the observable phases with the procedure mentioned above, we obtain the coupling functions shown in Fig. 2 (i) and (l). As one can see, these surfaces are drastically different from the original ones. Thus, even a small deviation from the linear relation between the observed and the theoretical phases can cause wrong estimations of the coupling functions. Since the observable phases are not uniquely defined this effect can lead to systematic errors in the reconstruction procedure of the coupling functions.

Kralemann et al. (2008) addressed this problem and suggested a transformation that is nothing else but the non-linear relation between the observable 

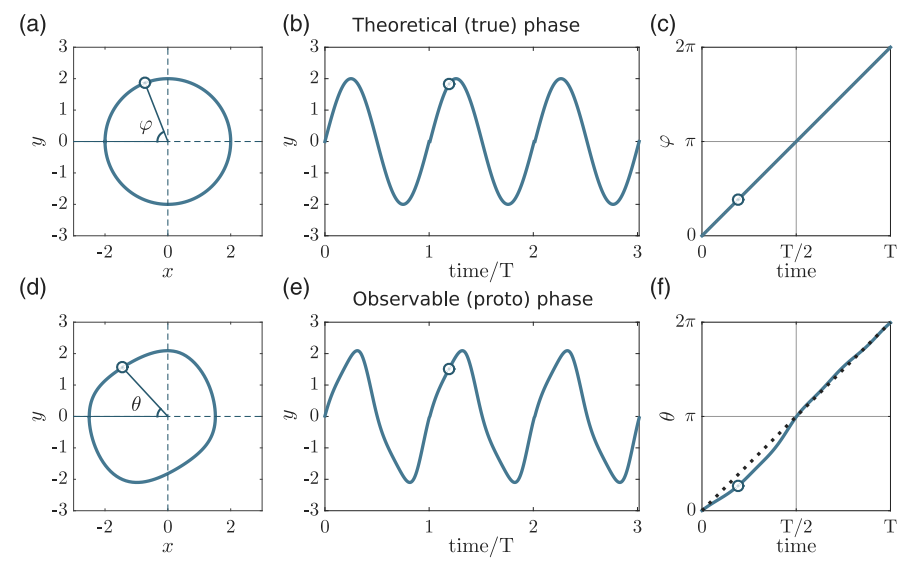

(e) Observable (proto) phase (f)
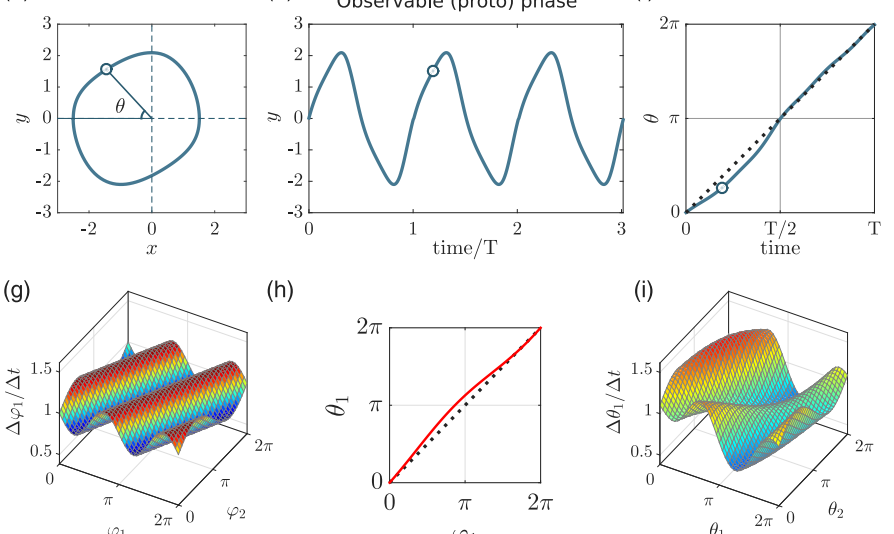

(h)
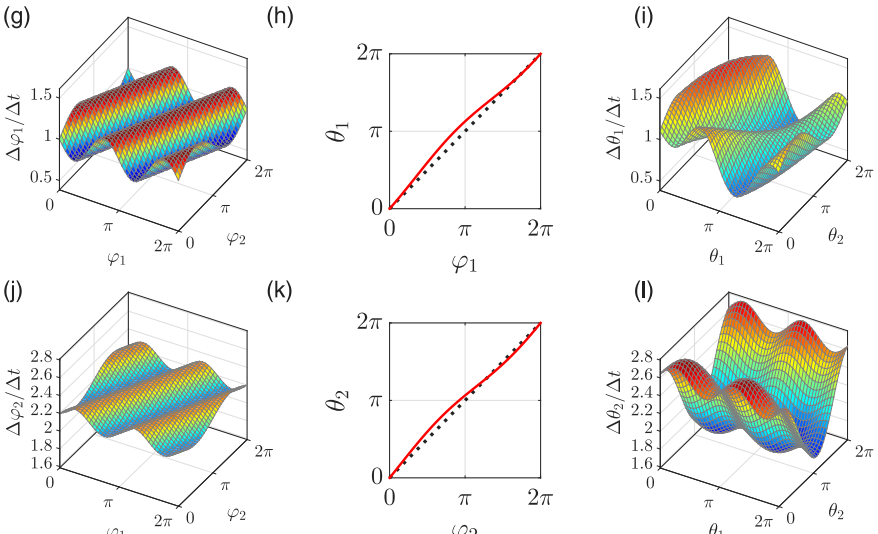

$(\mathrm{k})$
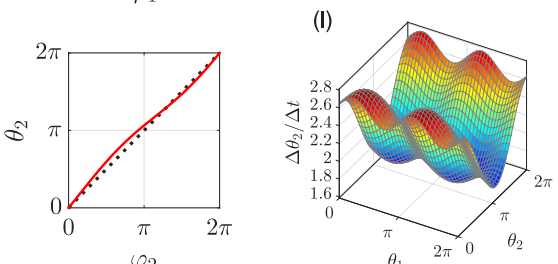

(m)

(n)

(o)
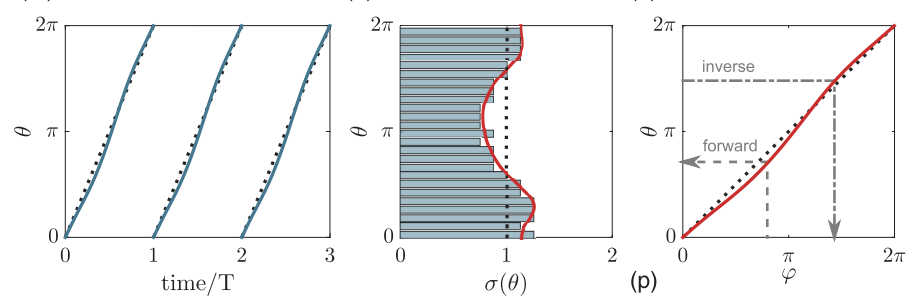

$$
\begin{aligned}
& \text { inverse transformation } \\
& \frac{d \varphi}{d t}=\frac{d \varphi}{d \theta} \frac{d \theta}{d t}=\sigma(\theta) \frac{d \theta}{d t}, \varphi=\Phi(\theta)=\int_{0}^{\theta} \sigma\left(\theta^{\prime}\right) d \theta^{\prime} \\
& \text { forward transformation } \\
& \frac{d \theta}{d t}=\frac{d \theta}{d \varphi} \frac{d \varphi}{d t}=\rho(\varphi) \frac{d \varphi}{d t}, \theta=\Theta(\varphi)=\int_{0}^{\varphi} \rho\left(\varphi^{\prime}\right) d \varphi^{\prime}
\end{aligned}
$$

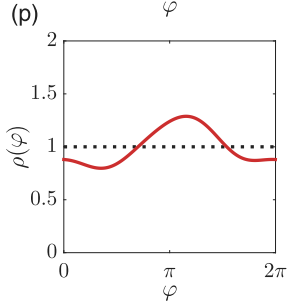

Figure 2: Theoretical phase, observable phase, and transformations between them. (a)-(c) The theoretical phase. (d)-(f) The observable phase. (g)-(l) The effect of non-linear relation between observable and theoretical phases. (m)-(p) Forward and inverse transformations and relations between them. See text for details. 
and the theoretical phases. This transformation can be found from the relation

$$
\frac{d \varphi}{d t}=\frac{d \varphi}{d \theta} \frac{d \theta}{d t}=\sigma(\theta) \frac{d \theta}{d t} .
$$

If we take into account that the theoretical phase $\varphi$ grows with constant speed $d \varphi / d t=\omega_{0}$, then

$$
\sigma(\theta)=\omega_{0} \frac{d t}{d \theta}(\theta)
$$

Thus, the function $\sigma(\theta)$ is inversely proportional to the velocity of $\theta$ and can be approximated by the probability distribution density of the observable phase $\theta$. This is demonstrated in Fig. $2(\mathrm{~m})$ and $(\mathrm{n})$. The time course of a non-linear growing observable phase $\theta$ is shown in Fig. $2(\mathrm{~m})$. The probability distribution density constructed from the time course gives us the approximation of $\sigma(\theta)$ (Fig. 2(n))

Using this fact one can find the function $\sigma(\theta)$ from the time series of the observable phase. The $2 \pi$-periodic function $\sigma(\theta)$ can be represented as a Fourier series

$$
\sigma(\theta)=\sum_{n} S_{n} e^{i n \theta},
$$

where the Fourier coefficients $S_{n}$ are

$$
S_{n}=\frac{1}{T} \int_{0}^{T} e^{-i n \tilde{\theta}(t)} d t
$$

where $\tilde{\theta}(t)$ is the time series of the observable phase measured over a time interval $T$. The normalization condition for the probability density requires $S_{0}=1$. As it was shown by Kralemann et al. (2008), the approximation Eq. (6) is also valid in the presence of a weak coupling $\left(q_{i j} \neq 0\right)$ if the time series is long enough to fulfill the ergodicity assumption.

By integrating $\sigma(\theta)$ with an additional condition $\varphi(\theta=0)=0$ we obtain

$$
\varphi=\Phi(\theta)=\int_{0}^{\theta} \sigma\left(\theta^{\prime}\right) d \theta^{\prime} .
$$

Here the function $\Phi(\theta)$ is the transformation function from the observable phase $\theta$ to the theoretical phase $\varphi$ (Fig. 2(o)). We call it the inverse transformation function (Kralemann et al. (2008) referred to it as the transformation from protophase to phase).

In the same manner we can define

$$
\rho(\varphi)=\frac{d \theta}{d \varphi}(\varphi)
$$

which also obeys the normalization condition $\int_{0}^{2 \pi} \rho(\varphi) d \varphi=1$. The integral of $\rho(\varphi)$ is a transformation from the theoretical phase $\varphi$ to the observable phase $\theta$, with the initial condition $\theta(\varphi=0)=0$ :

$$
\theta=\Theta(\varphi)=\int_{0}^{\varphi} \rho\left(\varphi^{\prime}\right) d \varphi^{\prime}
$$

We call this function the forward transformation function (Fig. 2(o) and (p)). It is obvious, that the inverse transformation function $\Phi(\theta)$ is an inverse of the forward transformation function $\Theta(\varphi)$. 
In the work presented here we implement this transformation in DCM for phase coupling. In the next section we explain the details of DCM in general and, more specifically, the version of DCM for phase coupling.

\subsection{Short description of DCM}

Dynamic Causal Modelling (DCM) was first presented in Friston et al. (2003) as a tool for the analysis of effective connectivity using fMRI data. More recently DCM was extended to EEG, MEG, LFPs, and functional near-infrared spectroscopy (fNIRS) (David et al., 2006; Chen et al., 2008; Stephan et al., 2008; Marreiros et al., 2008; Moran et al., 2009; Chen et al., 2012; Tak et al., 2015). DCM can be implemented with open-source software within the Statistical Parametric Mapping (SPM) software (Friston et al., 2007a).

Although there are different variants of DCM they all have a common general structure (Daunizeau et al., 2011). In Fig. 3 we present the structure of DCM as a diagram, where only components of DCM relevant to this work are shown. A more detailed overview of the DCM structure can be found in (Friston et al., 2003; Stephan et al., 2010; Daunizeau et al., 2011; van Wijk et al., 2018).

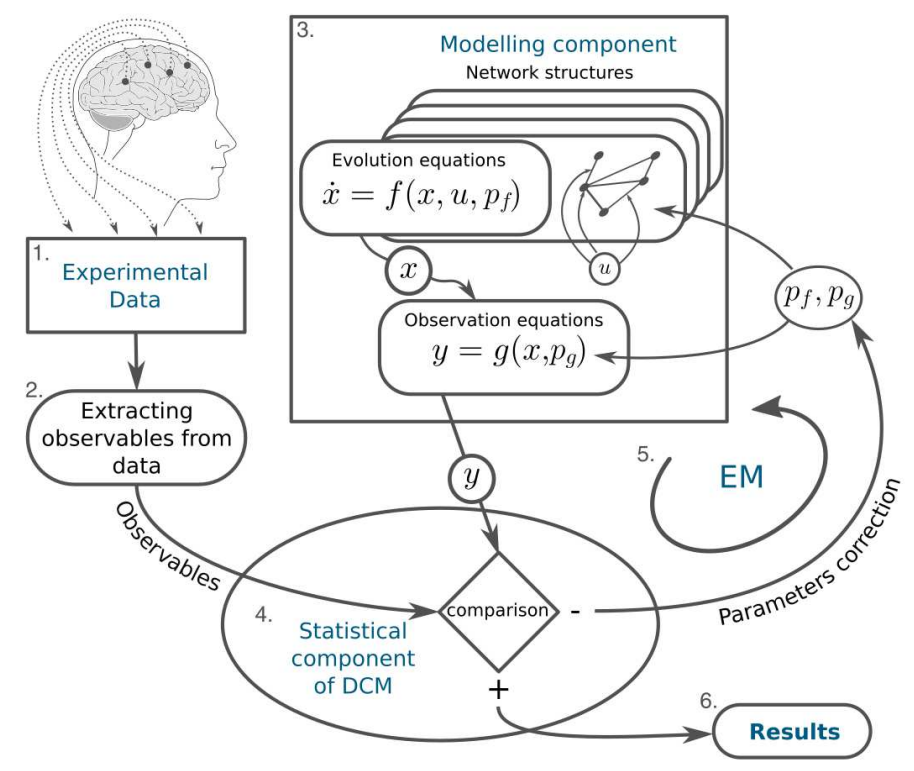

Figure 3: The general scheme of Dynamic Causal Modelling (DCM). See text for details.

The basic idea of DCM is to estimate the structure of neural interactions from experimentally measured brain activity by means of Bayesian inference. In this inferential framework, the brain is considered to be a non-linear dynamic system and represented as a directed graph with nodes and edges. The nodes correspond to source activities mediated by effective connectivity - the edges.

DCM can either be used to fit raw data or some pre-processed observables (Fig. 3(1) and (2)) that are chosen to make the inversion as efficient as possible. For example in fMRI, the observables are BOLD signals in selected regions of 
interest (Friston et al., 2003). For EEG/MEG, it is possible to fit the time series in sensor space - or treat reconstructed time series in source space as virtual electrodes (c.f., local field potentials). This is the approach taken in DCM for evoked responses (David et al., 2006) and in DCM for induced responses (Chen et al., 2008).

Later, the observables obtained from the experiment are "compared" with the observables generated by DCM by means of a non-linear dynamic system. Following Daunizeau et al. (2011) we call the part of DCM by which it generates the observed data the modelling component (Fig. 3(3)).

In the modelling component the source activity is defined by hidden (neural) states $(x)$. The hidden states change depending on the current state of the system, the experimental manipulations $(u)$ and the unknown evolution parameters $\left(p_{f}\right)$. This dependence is described by the evolution equations $\dot{x}=f\left(x, u, p_{f}\right)$. The evolution parameters $p_{f}$ and the structure of the evolution function $f$ entirely determine the nodes and the edges of the graph and how the edges influence the dynamics of the system. Moreover, DCM allows to consider several network structures (graphs) simultaneously and to chose the one which best fits the data, using Bayesian model comparison.

The systems' hidden states $(x)$ generated by the evolution equations are then mapped to observable variables $(y)$ using the observation equations $y=g\left(x, p_{g}\right)$, where $p_{g}$ are the unknown observation parameters (Fig. 3(3)).

The parameters of the evolution and observation equations are estimated in the statistical component of DCM (Fig. 3(4)) by means of Bayesian inversion (Friston et al., 2003; Daunizeau et al., 2011). This part of DCM has an identical scheme in all DCM variants with small modifications optimized for special cases. Under the Bayesian framework, the modelling component is used to derive a likelihood function, which determines how likely it is to observe a specific set of observations $y$ for a given set of parameter values $p=\left(p_{f}, p_{g}\right)$. The parameters of the modelling component $p$ are assumed to have a distribution $q(p)$ in the form of the Laplacian (i.e. Gaussian) approximation. Thus, the posterior density is $q(p)=N(\mu, \Sigma)$. The values of the conditional mean $\mu$ and the conditional covariance $\Sigma$ are updated in an iterative form using Variational Bayes, which resembles the Expectation-Maximisation (EM) algorithm ${ }^{2}$ (Friston et al., 2007b). Here, as a bound approximation to log model evidence, a free energy function $F(q, \lambda)$ is introduced ${ }^{3}$. In the E-step of the EM algorithm the free energy $F$ is maximized with respect to $q^{4}$ and in the M-step with respect to the precision parameter $\lambda$. The iteration continues until the free energy reaches its maximum. Using Bayesian model selection, DCM allows the comparison of different model structures (Penny et al., 2004). More details of the estimation scheme are given in (Friston, 2002; Friston et al., 2003, 2006).

For the sake of simplicity the statistical component of DCM is presented in the diagram in Fig. 3 as a conditional operation (4), which compares the experimentally obtained observables with the ones generated by the modelling component. The interactive update of the parameter values using the EM algorithm

\footnotetext{
${ }^{2}$ Although Bayesian model inversion in DCM is often described in terms of an EM scheme, it actually uses Variational Laplace; namely, variational updates under a fixed form (Gaussian) assumption about the posterior density. This is slightly more sophisticated than EM and enables uncertainty quantification about system and state noise levels.

${ }^{3}$ Negative free energy is a lower bound of the log model evidence.

${ }^{4}$ Maximizing the negative free energy.
} 
is displayed by the feedback-loop arrow from the statistical to the modelling component (Fig. 3(5)).

With the statistical component implemented in universal form, DCM provides a powerful tool for connectivity analyses in different biophysical models. The same type of measurements of brain activity can be analysed with different generative models, such as the two EEG/MEG extensions of DCM for evoked potentials (David et al., 2006) and for induced responses (Chen et al., 2008).

Penny and colleagues (2009) presented an extension of DCM to the analysis of phase-coupled data, where the generative model was based on weakly coupled phase oscillators. The network of weakly coupled phase oscillators was described by the following evolution equations

$$
\dot{\varphi}_{i}=\omega+\sum_{j}^{N_{r}} \Gamma_{i j}\left(\varphi_{i}-\varphi_{j}\right), i=1 \ldots N,
$$

where $\omega$ is the intrinsic frequency of the oscillators, $N_{r}$ is the number of regions and $\Gamma_{i j}(\varphi)$ is the phase interaction function. In the DCM for the phase-coupled data, the intrinsic frequencies of the oscillators were assumed to be the same and only intra-frequency couplings were considered. Therefore, the phase interaction function $\Gamma_{i j}(\varphi)$ was a function of the phase difference and was approximated by Fourier series as follows:

$$
\Gamma_{i j}(\varphi)=-\sum_{n=1}^{N_{s}} a_{i j n}^{s} \sin (n \varphi)+\sum_{n=1}^{N_{c}} a_{i j n}^{c} \cos (n \varphi)
$$

where $N_{s}$ and $N_{c}$ are the number of sine and cosine terms. The Fourier coefficients $a_{i j n}^{s}$ and $a_{i j n}^{c}$ are assumed to be positive and to change with respect to the modulatory inputs. The strength of the connection between any two regions is defined as the norms of the coefficients $\|a\|$. The observation equations in Penny et al. (2009) are defined to be linear and the observed time series in trial $k$ in region $i$ is the unwrapped phase variable $\varphi_{k i}$. Therefore, the initial values for the evolution equations are taken to be equal to the initial phases extracted from experimental measurements.

As we discussed in the previous section, the dynamics of phases measured in experiments differ from the theoretical ones, such as Eqs. (11) and (12). Neglecting this fact can lead to systematic errors in the estimation of the coupling functions. Thus, taking the modelling part of (Penny et al., 2009) as the basis, we here aim at resolving this problem by extending the modelling component of DCM. In the next section we present this extension.

\subsection{The extended modelling component}

The scheme of the DCM modelling component (Fig. 3(3)) itself suggests how the problem with the observable phase can be resolved. The non-linear relation between the observable and the theoretical phases can be directly implemented into the observation equations. Thus, the function $g$ represents the forward transformation function $\Theta(\varphi)$, which will be estimated together with the evolution equations by means of Bayesian inversion. The scheme of DCM with the extended modelling component is shown in Fig. 4. 
As one can see, the general scheme of DCM remains the same and the main changes concern the modelling component (Fig. 4(3)). However, non-linearity of the observation equation requires also a subsequent change in the statistical component. Therefore, and in contrast to the version of DCM in (Penny et al., 2009 ), in the extended version of DCM we used the version of Bayesian inversion for non-linear models with the Gauss-Newton method (Friston et al., 2007b).

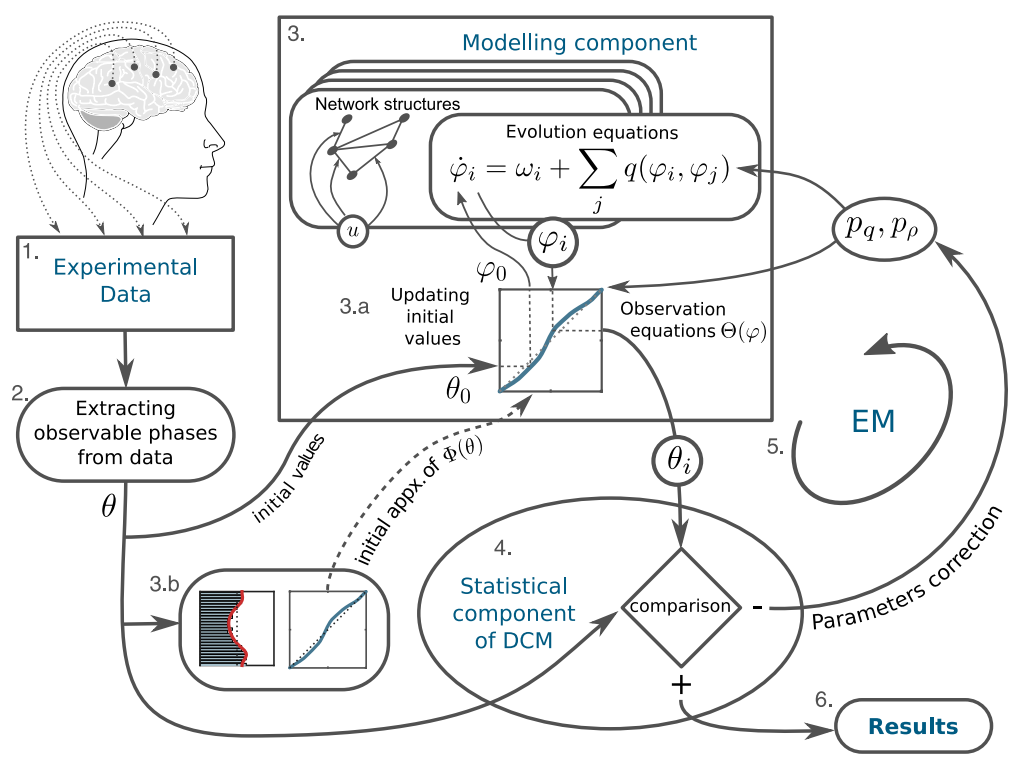

Figure 4: The scheme of the extended DCM for phase coupling. See text for details.

\subsubsection{Evolution and observation equations}

In our model, apart from introducing non-linear observation equations, we also extended the evolution equations to the case with different frequencies and a general form of the coupling function in order to allow detecting $n: m$ synchronization. The dynamical equation of the theoretical phase for a region $i$ is similar to Eq. (3)

$$
\dot{\varphi}_{i}=\omega_{i}+\sum_{j=1, j \neq i}^{N_{q}} q_{i j}\left(\varphi_{i}, \varphi_{j}\right)
$$

where $N_{q}$ is the number of regions and $\omega_{i}$ is the intrinsic frequency of the $i$-th oscillator. In contrast to Eq. (11), the intrinsic frequencies $\omega_{i}$ are generally different for every region. The periodic coupling functions $q_{i j}$ are approximated using two dimensional Fourier series:

$$
q_{i j}\left(\varphi_{i}, \varphi_{j}\right)=\sum_{n, m \neq 0} Q_{i j}^{(n, m)} e^{\mathrm{i}\left(n \varphi_{i}+m \varphi_{j}\right)}
$$

where $n$ and $m$ are integers, $Q_{i j}^{(n, m)}$ are the Fourier coefficients, which in general are complex numbers. We assume that the coupling functions are smooth and 
restrict the number of terms of the Fourier series to $N_{q}$. Thus, $n$ and $m$ changes from $-N_{q}$ to $N_{q}$, however, $n \neq 0$ and $m \neq 0$. We will discuss these conditions below.

The dynamics of the observable phase is then obtained by the forward transformation from the theoretical phase to the observable phase

$$
\dot{\theta}_{i}=\rho_{i}\left(\varphi_{i}\right) \dot{\varphi}_{i},
$$

where $\rho_{i}\left(\varphi_{i}\right)$ are defined as in Eq. (9). We approximate these $2 \pi$ periodic functions $\rho_{i}\left(\varphi_{i}\right)$ by Fourier series

$$
\rho_{i}\left(\varphi_{i}\right)=1+\sum_{k=1}^{N_{\rho}} P_{i}^{(k)} e^{\mathrm{i} k \varphi_{i}}
$$

where $P_{i}^{(k)}$ are complex Fourier coefficients. Note, that 1 on the right hand side of Eq. (16) ensures the normalization condition, i.e. $P_{i}^{(0)}=1$. Here we also assume the smoothness of the transformation functions and restrict the maximal number of terms of the Fourier series to $N_{\rho}$. Thus, $k$ changes from 1 to $N_{\rho}$.

We obtain the non-linear relation between observable and theoretical phases, i.e. the forward transformation function by integrating Eq. (15) (cf. Eq. (16) in (Kralemann et al., 2008)):

$$
\begin{aligned}
\theta_{i}=\Theta\left(\varphi_{i}\right) & =\int_{0}^{\varphi_{i}} \rho\left(\varphi^{\prime}\right) d \varphi^{\prime} \\
& =\varphi_{i}+\sum_{k \neq 0} \frac{P_{i}^{(k)}}{\mathrm{i} k}\left(e^{\mathrm{i} k \varphi_{i}}-1\right) .
\end{aligned}
$$

Since the transformation is only defined up to a constant, without loss of generality, we set here the initial condition as $\theta(\varphi=0)=0$.

In the extended DCM modelling part we use Eq. (14), Eq. (16), and Eq. (17) with real-valued Fourier coefficients for computational optimization purposes. For this, the complex coefficients $Q_{i j}^{(n, m)}$ are substituted by four real-valued coefficients $\left(p_{q}=\left\{a_{i j}^{(n, m)}, b_{i j}^{(n, m)}, c_{i j}^{(n, m)}, d_{i j}^{(n, m)}\right\}\right)$ and $P_{i}^{(k)}$ by two real-valued coefficients $\left(p_{\rho}=\left\{\alpha_{i}^{(k)}, \beta_{i}^{(k)}\right\}\right)$. The corresponding formulae are given in Appendix A.

\subsubsection{Assumptions of the model}

As we discussed in Section 2.3, for a successful reconstruction of the coupling function within the framework of coupled phase oscillators the coupling should be weak. Thus, we assume that the coefficients $Q_{i j}^{(n, m)}$ are small and the dynamics of the phase oscillators are far from the fully synchronized state.

The assumptions made for Eq. (14) originate from conditions that are important for a successful reconstruction (see Rosenblum and Pikovsky, 2001; Kralemann et al., 2008). The first one, $m \neq 0$, means that the Fourier series of the coupling function $q_{i j}\left(\varphi_{i}, \varphi_{j}\right)$ has no terms that depend on $\varphi_{i}$ only. The assumption is that these terms are only included in the transformation function $\rho(\varphi)$ (Eq. (16)), thereby giving a clear separation between $\rho(\varphi)$ and $\dot{\varphi}$ in Eq. (15). Moreover, the phase oscillator in Eq. (13) oscillates uniformly without coupling. 
The second condition $n \neq 0$ originates from the assumption that the coupling term depends oscillatorily on $\varphi_{j}$, i.e. on the forcing phase, which indicates that the driven oscillator is strongly synchronized with the forcing oscillator. If this is the case then we are unable to reconstruct the coupling function (Kralemann et al., 2008).

\subsubsection{Forward vs inverse transformations}

The main extension of our model is the introduction of the non-linear observation equations that reflect the transformation function. This approach differs from the one presented by Kralemann et al. (2008). In our model we find the forward transformation function (from $\varphi$ to $\theta$ ), whereas Kralemann et al. (2008) estimated the inverse transformation function (i.e. from $\theta$ to $\varphi$, see Fig. 2(o)). The advantage of our approach is that the parameters $p_{\rho}$ of the observation function are estimated together with the parameters $p_{q}$ of the coupling function using Bayesian inference (Fig. 4(3a)). This results in a better approximation of the transformation function $\Theta(\varphi)$.

However, there are drawbacks of this forward approach. First of all, the values of the parameters of the transformation function $p_{\rho}$ change with every estimation step. Since we only know the initial values of the observable phases $\theta_{0}$, the change of $p_{\rho}$ affects the choice of the initial conditions for Eq. (13). We resolve this problem by updating the initial values of $\varphi_{0}$ for Eq. (13) in every step of the Bayesian estimation by recalculating the inverse transformation function $\Phi(\theta)$ from the Fourier coefficients of the forward transformation function $P_{i}^{(k)}$ (Fig. 4(3a)).

Another not subtle issue is that the inverse transformation approach (Eq. (4) and Eq. (5)) provides a unique $\sigma(\theta)$ function, whereas the forward transformation (Eq. (9)) does not. The reason is that noisy data points can be fitted with different combinations of $\rho_{i}\left(\varphi_{i}\right)$ and $q_{i j}\left(\varphi_{i}, \varphi_{j}\right)$. To circumvent this problem, we estimate an initial approximation of $\sigma(\theta)$ (and of $\Phi(\theta)$, see Fig $4(3 \mathrm{~b})$ ) using the probability distribution method similar to the one discussed by Kralemann et al. (2008). Since the original method requires a long observation time period to approximate the probability distribution, we, therefore, developed a special method for the calculation of $\sigma(\theta)$ for a set of short trials (see Appendix B).

\subsection{Priors}

For the parameters of the evolution and observation equations we use the following priors

$$
\begin{aligned}
& p\left(Q_{i j}^{(n, m)}\right)=N\left(0, \sigma_{q i}\right), \\
& p\left(P_{i}^{(k)}\right)=N\left(P_{i 0}, \sigma_{\rho i}\right), \\
& p\left(f_{i}\right)=N\left(f_{i 0}, \sigma_{f i}\right),
\end{aligned}
$$

where $P_{i 0}$ are the values of the Fourier coefficients $P_{i}$ obtained from the initial approximation of $\sigma(\theta)$ and, respectively, of $\rho(\varphi)$ by the probability distribution method, $f_{i 0}$ is the mean value of the frequency after filtering the signal within the frequency band $f_{i 0} \pm \Delta_{f i}$. As Penny and colleagues (2009), we choose the variances of the parameters such that the instantaneous frequency is outside 
of the frequency band $f_{i 0} \pm \Delta_{f i}$ with the probability less than $p_{0}=0.001$. However, in our model this condition refers to the instantaneous frequency of the observable phase $\dot{\theta}$. Using Eq. (13), (15), and (16) we can write

$$
\dot{\theta}_{i} \sim\left(1+N\left(R_{i}, \sigma_{\rho i}\right)\right)\left(N\left(f_{0}, \sigma_{f i}\right)+N\left(0, \sigma_{q i}\right)\right),
$$

where $R_{i}=\left\|P_{i 0}\right\|$. Hence, the standard deviations of $f$ and $Q_{i j}$ increase $\left(1+R_{i}\right)$ times and the one of $P_{i}$ increases $f_{i 0}$ times. By requiring the condition above for any single term of this equation, we define the variances of the parameters as

$$
\begin{aligned}
\sigma_{f i}=\sigma_{q i} & =\left(\frac{\Delta_{i}-R f_{i 0}}{\left(1+R_{i}\right) z_{p}}\right)^{2}, \\
\sigma_{\rho i} & =\left(\frac{\Delta_{i}-R f_{i 0}}{f_{i 0} z_{p}}\right)^{2},
\end{aligned}
$$

where $z_{p}$ is the value of the quantile (or inverse cumulative distribution) function at $p_{0} / 2=0.0005$.

\section{Results}

In this section we applied the extended DCM for phase coupling on synthetic data generated by models with known parameters. We performed two tests on the basis of two different models ${ }^{5}$. The first one is a model of weakly coupled phase oscillators. The second is a neural mass model.

\subsection{Coupled phase oscillators}

In this test we aim at demonstrating the necessity of considering the non-linear relation between the observable and theoretical phases. Therefore, we reconstructed the coupling functions of the same system using DCM for phase coupling developed by Penny et al. (2009) and its extended version derived in the previous section. In other words, we analysed the data using a standard DCM based upon the original neuronal model (Eqs. (11) and (12) further denoted as DCM PC), and an extended DCM (Eqs. (13),(14), and (17) further denoted as eDCM PC) that allows for different intrinsic frequencies at each node or source. This extended DCM PC characterises effective connectivity in terms of a two-dimensional phase coupling function and, crucially, allows for phenomena such as m:n phase coupling and can detect the non-linear relation between the observable and theoretical phases.

As a generative model we took a model of uni-directionally weakly coupled phase oscillators

$$
\begin{aligned}
& \dot{\varphi}_{1}=\omega_{1}+\eta_{1}(t), \\
& \dot{\varphi}_{2}=\omega_{2}+a \sin \left(\varphi_{2}-\varphi_{1}\right)+\eta_{2}(t),
\end{aligned}
$$

where $a$ is the coupling strength, $\omega_{1}=\omega_{2}$ (see Fig. 5), and $\eta_{1,2}(t)$ are normally distributed noises. We chose this form of the coupling function since it is used in

\footnotetext{
${ }^{5}$ All simulation codes can be found here https://gitlab.com/azayeld/edcmpc/
} 
DCM PC and allowed us to compare the two modelling components. A correct reconstruction of a uni-directional coupling implies a zero coupling strength for the first oscillator $\left(A_{1,2}=0\right)$ and a non-zero one for the second $\left(A_{2,1}=a\right)$.

Using Eq. (22) we generated two different data sets: one containing the theoretical phases and one the observable phases. The observable phases were obtained by using the forward transformation function of the form Eq. (17) (see Fig. 5).

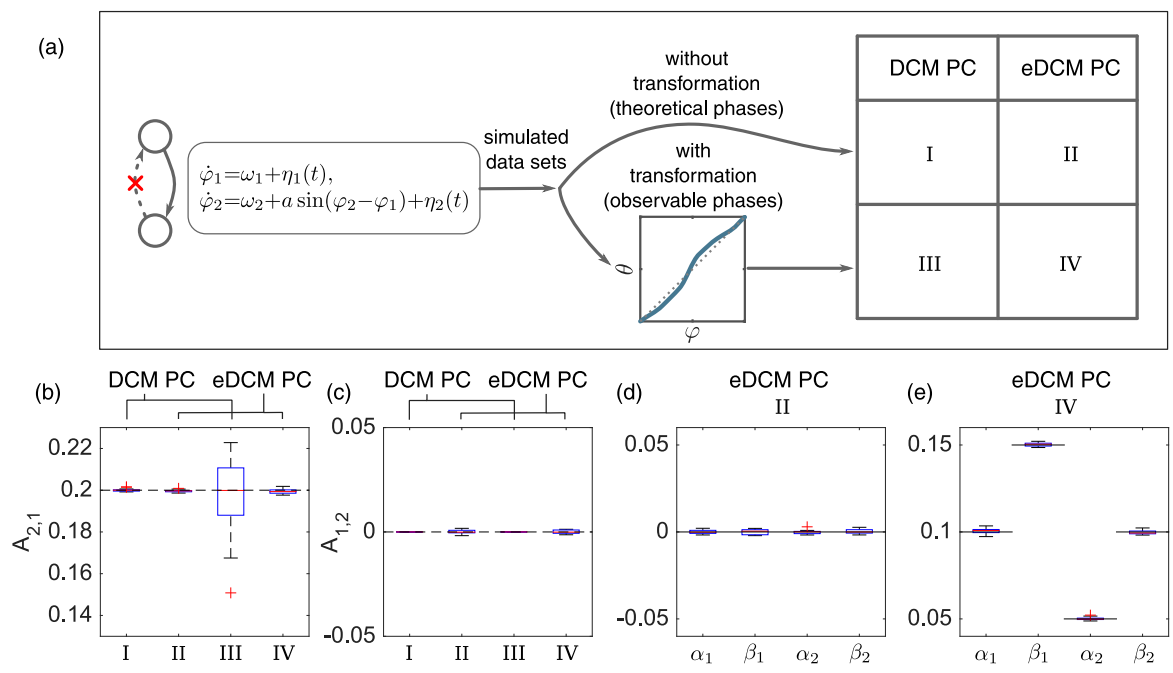

Figure 5: The results of the reconstruction of the coupling strength of two unidirectionally coupled phase oscillators using DCM for phase coupling (DCM PC) and the extended version of it (eDCM PC). (a) Two types of data sets were generated: with and without transformation. Thus, four cases denoted with the numbers I, II, III, and IV were analysed in total. (b) and (c) show the results of the reconstructed coupling strengths of the second and the first oscillators, respectively. (d) and (e) show the results of the reconstruction of the Fourier coefficients of the transformation function using eDCM PC for the cases II and IV, respectively.

The coupling functions are reconstructed in DCM PC as real-valued Fourier coefficients. Therefore, within the framework of DCM PC the model Eq. (22) is represented as the coefficients of Eqs. (11) and (12):

$$
a_{121}^{s}=A_{1,2}=a, a_{211}^{s}=A_{2,1}=0 .
$$

All other coefficients are zero $\left(a_{i j n}^{c}=0\right)$. For the eDCM PC the model Eq. (22) is represented as the coefficients of Eq. (13) and Eq. (14), i.e. of Eq. (23) (A):

$$
\begin{aligned}
& b_{12}^{(11)}=-c_{12}^{(11)}=A_{1,2}=a, \\
& b_{21}^{(11)}=c_{21}^{(11)}=A_{2,1}=0,
\end{aligned}
$$

and all other coefficients are zero:

$$
\begin{aligned}
& a_{12}^{(11)}=b_{12}^{(11)}=0 \\
& a_{21}^{(11)}=b_{21}^{(11)}=0 .
\end{aligned}
$$


We generated 15 synthetic data sets with 20 trials each by integrating Eq. (22) with random initial conditions. For integration we used the weak converging second order Runge-Kutta type method for stochastic differential equations (Platen, 1987; Honeycutt, 1992; Higham., 2001; Sauer, 2012). During the integration of the system Eq. (22) we applied normally distributed noises $\left(\eta_{1}(t)\right.$ and $\left.\eta_{2}(t)\right)$, each with amplitude of 0.05 and standard deviation of 0.1 to both oscillators. The parameter values were $\omega_{1}=\omega_{2}=1.0 \mathrm{rad} / \mathrm{s}$ and $a=0.2$. Every trial lasted $4 \mathrm{~s}$ and had 80 time points. The real-valued Fourier coefficients of the transformation (Eq. (16) and, respectively, of Eq. (24)) were chosen as $\alpha_{1}^{(1)}=0.1, \beta_{1}^{(1)}=0.15, \alpha_{2}^{(1)}=0.05, \beta_{2}^{(1)}=0.1$. As mentioned above, DCM $\mathrm{PC}$ and eDCM PC were applied to two types of data sets - with and without transformation - to reconstruct the coupling functions. Therefore, four cases were analysed in total (see Fig. 5(a)). They are denoted with the numbers I, II, III, and IV as shown in the Figure.

As a result of the reconstruction, for every data set we obtained the values of the coupling strengths $A_{2,1}$ and $A_{1,2}$, presented as box plots in Fig. 5 (b) and (c). For DCM PC the coupling strengths are the values of $a_{i j 1}^{s}$, whereas for eDCM PC they are $b_{i j}^{(11)}$ and $-c_{i j}^{(11)}$.

From the data set of theoretical phases (the cases I and II), both versions of DCM reconstructed the correct values of the coupling strength $A_{2,1}$ (Fig. 5(b)). Moreover, the coupling strength $A_{1,2}$ was also estimated as zero by eDCM PC (Fig. 5 (c), the cases II and IV). For DCM PC we set the network structure as uni-directional. Therefore, in Fig. 5(c) no values of $A_{1,2}$ are shown for the cases I and III.

DCM PC is designed for this case, namely to reconstruct the models Eq. (22) from the theoretical phases. The extended DCM PC, however, is designed for general cases to reconstruct the network structure from observable phases and without assumptions on the transformation function (presence or absence of it). Nevertheless, for the data set of the theoretical phases eDCM PC estimates the values of the Fourier coefficients of the transformation function as zero, i.e. no transformation present (Fig. 5(d)).

Crucial results were obtained for the case III: for observable phases, DCM $\mathrm{PC}$ gave quite variable results for the different data sets (Fig. 5(b)). By contrast, eDCM PC estimated the correct values of the coupling strength (the case IV in Fig. 5(b)). Moreover, the values of the Fourier coefficients of the transformation function were also correctly estimated by eDCM PC (Fig. 5(e)).

\subsection{Neural mass model}

In this section we present the results of the second test. In contrast to the test presented in the previous section, we generated a synthetic data set that is a good approximation of an EEG signal based on the neural mass model developed by Jansen and Rit (1995). The system of equations and the parameter values of the neural mass model are given in Appendix C. This model was used by David and colleagues (2004) to evaluate different connectivity measures. Similarly, Vindiola et al. (2014) used two non-linearly coupled neural mass models of this type to analyse different EEG phase synchronization measures. Moreover, the neural-mass model of Jansen-Rit is used in the modelling component of the DCM for evoked responses (David et al., 2006). 

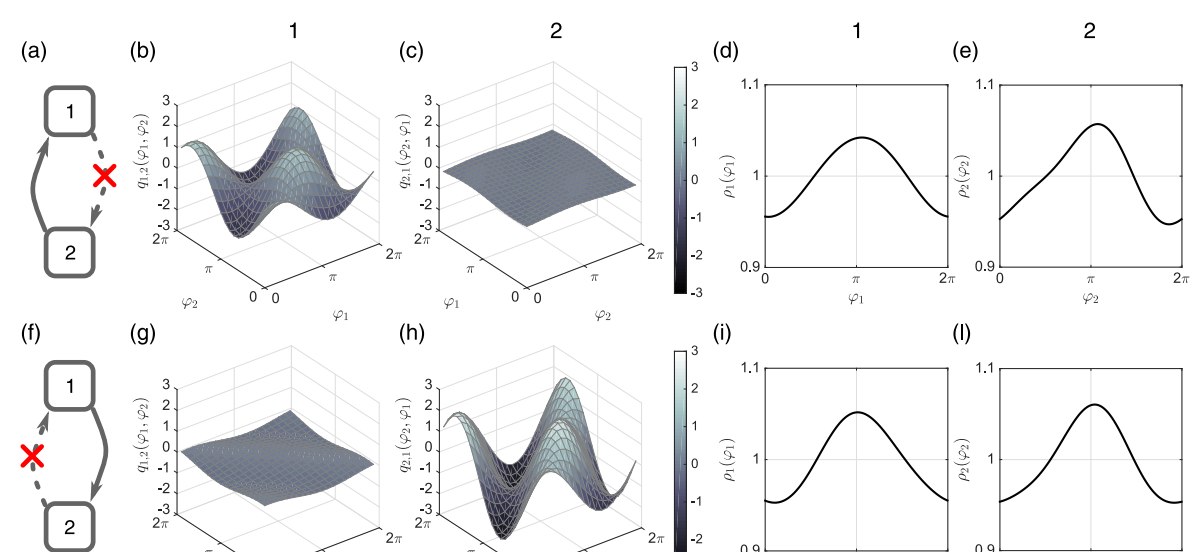

(g)

(h)

(i)
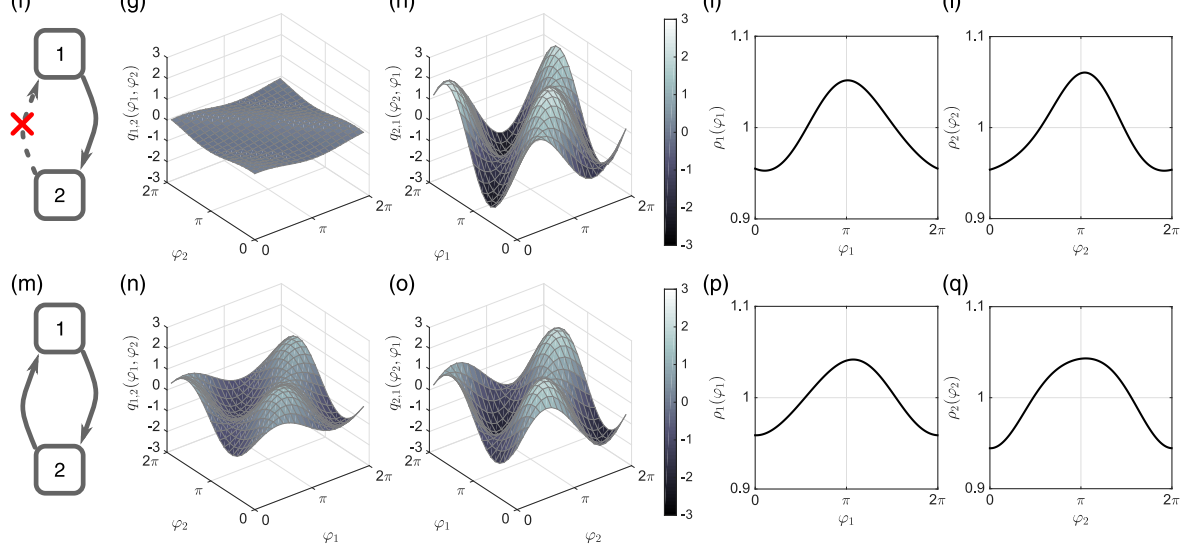

Figure 6: The results of the reconstruction of the coupling functions for different coupling configurations between two neural mass models.

We considered two coupled regions with three coupling cases as shown in Fig. 6 (a), (f), and (m). The parameters of the neural mass models of the different regions were set to generate oscillatory signals with different frequencies $\left(f_{1}=9.233 \mathrm{~Hz}\right.$ and $\left.f_{2}=16.160 \mathrm{~Hz}\right)$. Examples of the generated time series are presented in Appendix D. These synthetic EEG signals were then used to obtain the observable phases by means of the Hilbert transformation. From these data sets of observable phases for different coupling cases we then estimated the coupling functions between these regions using the extended DCM PC. In all cases, the coupling parameters of DCM were set to a bidirectional coupling. Thereby, we wanted the extended DCM PC to detect the absence of one of the couplings in the first (a) and the second case (f) in Fig. 6.

For every coupling case, we generated 15 trials with a temporary activated coupling to simulate the transient synchronization. Every trial started with random initial conditions and with deactivated coupling between the regions. After $0.5 \mathrm{~s}$ of the transient time period the couplings were activated for $0.3 \mathrm{~s}$ with a given coupling configuration (Fig. 6 (a), (f), or (m)). Then the system was simulated again for another 0.5 seconds with deactivated coupling (see Fig. 8 in Appendix D). As in the previous section, for the numerical integration of the coupled Jansen-Rit neural mass models, we used the weak converging second order Runge-Kutta type method for stochastic differential equations (Platen, 1987; Honeycutt, 1992; Higham., 2001; Sauer, 2012).

Within the parameter ranges used in our simulations, the system of two coupled neural mass models has several stable limit cycles, i.e. is multistable (Spiegler et al., 2010; Ahmadizadeh et al., 2018). Therefore, we used a balanced 
input to the regions to ensure that the system remains in the vicinity of the same limit cycle. Without coupling, the regions received a noisy input with a given mean and standard deviation (see C). When the coupling was activated, the balanced input was achieved by setting the constant part and the variance of the variable part of the overall input to the regions to remain the same. Thus, the mean and the standard deviation of the input noise of one region were reduced with respect to the mean and standard deviation of the input from the other region. This scheme is similar to the one used by David and Friston (2003).

After extracting the observable phases with the Hilbert transformation we cut out the transient parts of the trials. Thus, only the time period with activated coupling was used to reconstruct the coupling functions.

To assess the number of terms of the Fourier series in Eqs. (9) and (14), we first performed a spectral analysis on the data sets (see Appendix E). According to the results of this analysis, we took $N_{q}=1$ in Eq. (14) and $N_{\rho}=2$ in Eq. (9) as an approximation of the coupling function $q_{i j}$ and the forward transformation function $\rho_{i}$. Therefore, for every region four real-valued coefficients of the coupling function (Eq. (23)) and four real-valued coefficients for the transformation function (Eq. (24)) were estimated.

The results of the reconstruction are shown in Fig. 6, where the panels are lined-up with respect to the coupling configurations shown in (a), (f), and (m). The coupling functions are presented as surfaces and the columns correspond to the regions: (b), (g), (n) are the coupling functions for the first region and (c), (h), (o) the ones for the second region (cf. the numbers above the panels in Fig. 6), respectively. Similarly, the forward transformation functions $\rho_{1}\left(\varphi_{1}\right)$ and $\rho_{2}\left(\varphi_{2}\right)$ are presented for the different regions in columns: (d), (i), (p) are for the first region and (e), (l), (q) for the second region.

As can be seen, the extended DCM for phase coupling was able to detect the directionality of the coupling: for the cases when the connections were absent (Fig. 6 (a) and (f)) the coupling functions for the second (c) and for the first oscillators (g) were almost zero. By contrast, for the existing connections the coupling functions were not zero (Fig. 6 (b), (h), (n) and (o)). Moreover, the form of the reconstructed coupling functions suggests that $q_{i j}\left(\varphi_{i}, \varphi_{j}\right)$ is not a function of phase differences $\varphi_{j}-\varphi_{i}$ : the surface is not a wave function along the diagonal $\varphi_{j}-\varphi_{i}$. However, for a stronger coupling strength the function $q_{i j}\left(\varphi_{i}, \varphi_{j}\right)$ approached $\sin \left(\varphi_{j}-\varphi_{i}\right)$ (cf. Fig. 6 (h) and (o)), which is the form of the coupling assumed in the theory of synchronization of weakly coupled oscillators and in the DCM for phase coupled data (Penny et al., 2009) (see Eq. (12))

In all considered coupling configurations and for both regions the forward transformation functions were not constant $(\rho(\varphi) \neq 1$ ) (Fig. 6 (d), (i), (p), (e), (l), and (q)). This indicates that the oscillations generated by the neural mass model have non-sinusoidal form even without coupling (Fig. 6 (e) and (i)). Similar conclusions can be made from the time course of the derivatives of the observable phases (see Fig. 8 and the description in Appendix D). Furthermore, $\rho(\varphi)$ changed with respect to the coupling, i.e. the coupling changed the form of the limit cycle. 


\section{Discussion}

In this work we extended the modelling component of DCM for phase-coupled data so that it can work with phase variables obtained from experimentally measured observables.

DCM for phase-coupled data with the extended modelling component presented in this work differs from Penny et al. (2009) in several critical aspects. First of all, in the evolution equations Eq. (13)different natural frequencies of the oscillators are assumed. Therefore, the coupling function $q_{i j}$ is approximated by two dimensional Fourier series, thereby making it possible to apply the model to analyse cross-frequency couplings and consider the $n: m$ phase locking cases.

The main extension is related to the transformation function implemented as non-linear observation equations in the modelling component. In contrast to Kralemann et al. (2008), where the observable phases are transformed to the theoretical ones, our approach does the opposite: we estimate the forward transformation function, which finds the observable phases from the theoretical ones. This difference results in a remarkable effect when applied to real data. The inverse transformation (the transformation from protophase to phase) introduced by Kralemann et al. (2008) estimates from arbitrarily defined observable phases a unique phase variable due to a strict condition on the distribution of the true (theoretical) phases. By contrast, the forward transformation has no such explicit condition. Therefore, no unique fitting solution could occur for the observable phases obtained from the noisy data. In the Bayesian framework implemented in DCM, this means that the free energy function has several local maxima. However, only one maximum of the free energy, or equivalently, only a specific set of parameter values satisfies the condition on the distribution of the true (theoretical) phases. In our extension we solve this issue by calculating an initial approximation of the distribution density of the observable phases, i.e. the function $\sigma(\theta)$. Using this initial approximation, we define the initial priors for the parameters of the forward transformation function $\rho(\varphi)$.

Another difference of our approach compared to Kralemann et al. (2008) is that the parameter values of the transformation function are estimated together with the parameter values of the coupling function. Kralemann and colleagues (2008) also presented a method with a simultaneous estimation of the parameter values for two oscillators by means of solving the nonlinear equation of the Fourier coefficients. The convergence condition there was the minimum of the mean square error. This is equivalent to the condition of the maximum likelihood under the assumption that the observables are normally distributed. This differs from the Bayesian approach used in DCM (Friston, 2002; Friston et al., 2002a,b, 2007b), where the assumption is that not only the observables, but also the parameters are random variables with Gaussian distribution. Moreover, the Bayesian model comparison allows to compare different network configurations using the Bayesian model selection (David and Friston, 2003).

In this work we have demonstrated that it is necessary to distinguish the observable phase from the theoretical one. In the reconstruction of known coupling functions of two uni-directionally coupled phase oscillators (see Fig. 5) the obtained values of the coupling strengths were estimated wrongly if the observable phases were considered as theoretical ones (Fig. 5(b) III). Certainly, this error occurred due to the definition of the coupling function as a function of phase differences in the original DCM for phase coupling (see Eq. (11)). How- 
ever, even if the coupling function is defined in a general form, the reconstructed function can be estimated only with a significant error, as shown in Fig. 2(i) and (l).

The example using the neural mass model, which simulates real experimental measurements, revealed that the observables of the system, and, respectively, the observable phases have non-linear distributions (Figs. 6 and 8). Moreover, it was shown that the distribution is non-linear even without coupling $\left(\rho_{i}\left(\varphi_{i}\right) \neq\right.$ const for (e) and (i) in Fig. 6) and that the coupling changes the form of the limit cycle. The latter aspect is not considered in the framework of weakly coupled oscillators, since it does not account for changes in the amplitude. However, by introducing the transformation function we can eliminate the effect of these changes of the limit cycles.

A wrong estimation of the coupling strength is caused by the fact that generally the observable phase itself is distributed non-uniformly, which is unrelated to the coupling. Recent works addressed this problem. For example Scheffer-Teixeira and Tort (2016) showed that waveform asymmetry and frequency harmonics may generate artificial $n: m$ phase-locking. Belluscio and colleagues (2012) considered the asymmetry of hippocampal theta waves and suggested a method to define phase by identifying the waveform-based phase of spiking to make the estimation of cross-frequency phase-phase coupling more reliable.

The non-uniform distribution of the observed phase variables is an issue not only in cross-frequency phase coupling (CFC), but also in measuring the connectivity by means of the phase-to-amplitude coupling (PAC). Lozano-Soldevilla et al. (2016) showed that non-sinusoidal wave morphologies of neurophysiological oscillations can produce spurious couplings between amplitude and phase. Similarly, van Driel, Cox, and Cohen (2015) demonstrated that non-uniform phase angle distributions can, under specific circumstances, produce statistical errors and uninterpretable results when using PAC. The authors suggested a new measure called debiased PAC (dPAC). The logic behind this measure is to shift the phase angle distribution prior to computing PAC such that the distribution has a mean of zero, thereby effectively "uniformizing" the distribution of the phase angles. In order to solve this issue it might be necessary to introduce a transformation function for PAC, in a manner similar to the coupled phase oscillators discussed by Kralemann et al. (2008) and used in the current work.

The extension of DCM for phase coupling can be applied in different biological systems. The main condition is weakness of the coupling. The weak coupling can be visualized as a wide distribution of the data points on the phasephase surface (torus, see Fig. 1 (b)). This can be easily achieved in the case of transient synchronization.

The application of the results of this work is not restricted to measurements of brain activity, such as EEG, MEG, LFP, and iEEG only. The system of interest can be any network of weakly coupled self-sustained oscillators. Moreover, the activity of the network could also stem from different origins. For example, in experiments with a periodic input one could consider the external drive as an oscillator and analyse its influences on individual nodes of a network. Therefore, the extended DCM for phase-coupled data might be used to study the interaction in various classes of networks of weakly coupled phase oscillators.

The extension to DCM presented here should be used whenever there is a distinct asymmetry of the distribution of the measured phase. Moreover, as it 
was shown in the simulation of the coupled neural mass models, in the case of strong coupling the phase interaction function approaches a periodic function of the phase differences (Eq. (12)). Therefore, it is preferable to use DCM for phase-coupled data presented by Penny and colleagues (2009) for uniform distributed observable phase $(\rho(\varphi) \approx 1)$ and for strong coupling.

Despite the shown advantages, the new modelling component presented in this work has also disadvantages. The simultaneous estimation of the parameters of the coupling and the transformation function increases the number of parameters per region, which reduces the speed of their estimation procedure. Moreover, since the values of the parameters of the transformation function are calculated in every estimation step, it requires an additional operation: update of the initial values of the theoretical phases. Optimization of the speed of the estimation procedure and of the Bayesian inference for this specific type of model is a topic of future work.

\section{Acknowledgments}

1. This work was funded by the University of Cologne Emerging Group Initiative (CONNECT) within the framework of the Institutional Strategy of the University of Cologne and the German Excellence Initiative.

2. SD gratefully acknowledges additional support from the German Research Foundation (DA1953/5-2).

3. We would like to thank T.I.Tóth for useful discussions of the manuscript.

\section{A The model with real-valued Fourier coefficients}

In the numerical simulations we present Eqs. (14), (16), and (17) with realvalued Fourier coefficients for computational expediency.

The coupling function between the region $i$ and $j$ Eq. (14) is given by

$$
\begin{aligned}
q_{i j}\left(\varphi_{i}, \varphi_{j}\right)= & \sum_{n=1}^{N_{q}} \sum_{m=1}^{N_{q}}\left[a_{i j}^{(n, m)} \cos \left(n \varphi_{i}\right) \cos \left(m \varphi_{j}\right)+b_{i j}^{(n, m)} \cos \left(n \varphi_{i}\right) \sin \left(m \varphi_{j}\right)\right. \\
& \left.\quad+c_{i j}^{(n, m)} \sin \left(n \varphi_{i}\right) \cos \left(m \varphi_{j}\right)+d_{i j}^{(n, m)} \sin \left(n \varphi_{i}\right) \sin \left(m \varphi_{j}\right)\right]
\end{aligned}
$$

where $a_{i j}^{(n, m)}, b_{i j}^{(n, m)}, c_{i j}^{(n, m)}, d_{i j}^{(n, m)}$ are the real-valued Fourier coefficients. According to the assumptions $(n \neq 0$ and $m \neq 0)$ made in Section 2.6.2, the upper indexes of these coefficients change from 1 to $N_{q}$. Thus, every coupling coefficient (matrix) between any pair of regions $i$ and $j$ is of size $N_{q} \times N_{q}$.

The forward transformation function Eq. (16) with real-valued Fourier coefficients reads

$$
\rho\left(\varphi_{i}\right)=1+\sum_{k=1}^{N_{\rho}}\left[\alpha_{i}^{(k)} \cos \left(n \varphi_{i}\right)+\beta_{i}^{(k)} \sin \left(n \varphi_{i}\right)\right]
$$


where $\alpha_{i}^{(k)}$ and $\beta_{i}^{(k)}$ are the Fourier coefficients, each of the size $N_{q}$ for every region. The forward transformation function (Eq. (17)) is then

$$
\theta_{i}=\varphi_{i}+\sum_{k=1}^{N_{\rho}} \frac{1}{k}\left[\alpha_{i}^{(k)} \sin \left(n \varphi_{i}\right)-\beta_{i}^{(k)} \cos \left(n \varphi_{i}\right)+\beta_{i}^{(k)}\right] .
$$

\section{B Calculation of the probability distribution den- sity for short trials}

The probability distribution density of a $2 \pi$ periodic phase variable can be estimated with conventional methods if the system is observed for a long period of time. For a set of short period observations, such as short trials, the estimation of the distribution is accurate only if the observation time has full periods as shown in Fig. 7: for linearly growing phase $\varphi$ (a) the distribution density is uniform (b), whereas for non-linearly growing phase $\theta$ (f) it is not (g). If we observe no full periods of oscillations, as shown in Figs. 7 (c) and (h), then the estimated distribution appears to be wrong ((d) and (i)). From the distribution
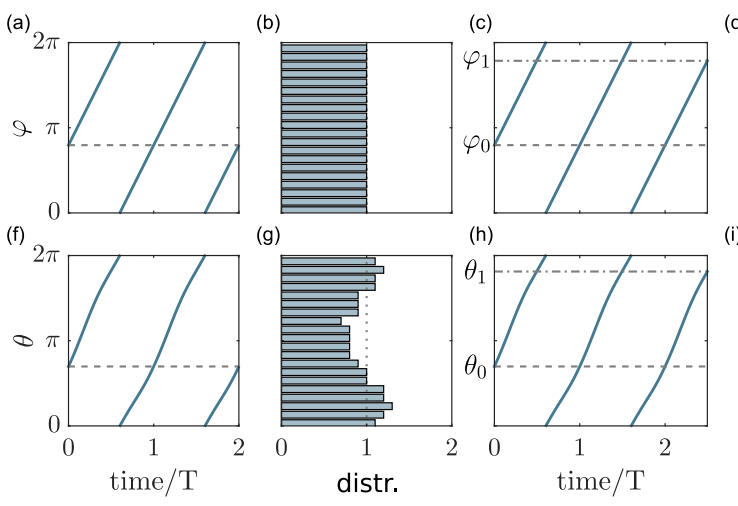

(d)

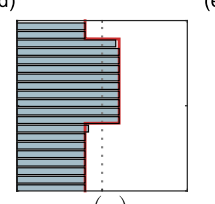

(e)

(h)

(i)

$\eta(\varphi)$

(j)
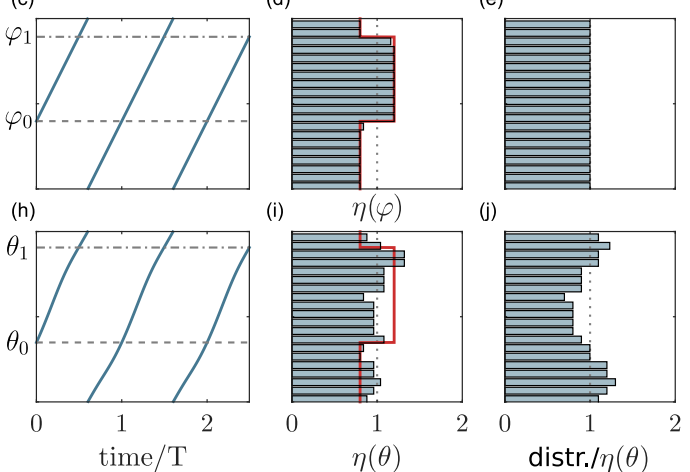

Figure 7: Estimation of the probability distribution density for short trials.

density of the linearly growing phase $\varphi$ we can calculate its envelope $\eta(\varphi)$ (red solid line in Fig. 7 (d)) as:

$$
\eta(\varphi)= \begin{cases}\frac{k+1}{k+\left(\varphi_{1}-\varphi_{0}\right) /(2 \pi)}, & \text { if } \varphi \in\left(\varphi_{0}, \varphi_{1}\right], \\ \frac{k+\left(\varphi_{1}-\varphi_{0}\right) /(2 \pi)}{}, & \text { otherwise }\end{cases}
$$

where $\varphi_{0}$ and $\varphi_{1}$ are the initial and the final values of $\varphi$ in the considered time course, respectively. By dividing the distritubtion density of $\varphi$ (Fig. 7(d)) by $\eta(\varphi)$ we obtain the correct distribution density as shown in Fig. 7 (e). Similarly, we can calculate $\eta(\theta)$ for the non-linearly growing phase $\theta$ (Figs. 7(h) and (i)):

$$
\eta(\theta)= \begin{cases}\frac{k+1}{k+\left(\theta_{1}-\theta_{0}\right) / 2 \pi}, & \text { if } \theta \in\left(\theta_{0}, \theta_{1}\right] \\ \frac{k}{k+\left(\theta_{1}-\theta_{0}\right) / 2 \pi}, & \text { otherwise, }\end{cases}
$$

where $\theta_{0}$ and $\theta_{1}$ are the initial and the final values of $\theta$ as shown in Fig. 7(h). After dividing the distribution density of the non-linearly growing phase $\theta$ 
(Fig. 7(i)) by $\eta(\theta)$ the resulting distribution density is the correct one (compare $(\mathrm{g})$ and $(\mathrm{j})$ in Fig. 7).

The presented method is valid if the derivative of the phase is always positive. This is equivalent to the condition that requires a weak coupling $\left\|q_{i, j}\right\| \ll 1$ and $\left\|P^{(k)}\right\| \ll 1$ in Eq. (17).

\section{Coupled Jansen-Rit neural mass model}

Every region in the Jansen-Rit neural mass model consists of three blocks - two excitatory and one inhibitory. The regions are connected via delay blocks. The system of equations for region $i$ reads

$$
\begin{aligned}
\dot{y}_{i}^{(0)} & =z_{i}^{(0)} \\
\dot{z}_{i}^{(0)} & =A_{i} a_{i} S\left(y_{i}^{(1)}-y_{i}^{(2)}\right)-2 a_{i} z_{i}^{(0)}-a_{i}^{2} y_{i}^{(0)}, \\
\dot{y}_{i}^{(1)} & =z_{i}^{(1)} \\
\dot{z}_{i}^{(1)} & =A_{i} a_{i}\left(p_{i}(t)+C_{2} S\left(C_{1} y_{i}^{(0)}\right)+\sum_{j=1, j \neq i}^{N_{s}} K_{j} y_{j}^{(d)}\right) \\
& -2 a_{i} z_{i}^{(1)}-a_{i}^{2} y_{i}^{(1)}, \\
\dot{y}_{i}^{(2)} & =z_{i}^{(2)} \\
\dot{z}_{i}^{(2)} & =B_{i} b_{i}\left(C_{4} S\left(C_{3} y_{i}^{(0)}\right)\right)-2 b_{i} z_{i}^{(2)}-b_{i}^{2} y_{i}^{(2)}, \\
\dot{y}_{i}^{(d)} & =z_{i}^{(d)}, \\
\dot{z}_{i}^{(d)} & =A_{i} a^{(d)} S\left(y_{i}^{(1)}-y_{i}^{(2)}\right)-2 a^{(d)} z_{i}^{(d)}-\left(a^{(d)}\right)^{2} y_{i}^{(d)},
\end{aligned}
$$

where the lower indices correspond to the regions, and the upper ones to the different blocks $((0)$ and (1) to excitatory blocks, (2) to inhibitory blocks, $(d)$ to delay blocks), $S(x)$ is a sigmoid function

$$
S(x)=\frac{2 e_{0}}{1+\exp \left(r\left(V_{0}-x\right)\right)} .
$$

We simulated two coupled regions $\left(N_{s}=2\right)$. The output signals of the regions are $Y_{1}=y_{1}^{(1)}-y_{1}^{(2)}$ and $Y_{2}=y_{2}^{(1)}-y_{2}^{(2)}$. According to (Jansen and Rit, 1995) we use the following relation between the parameters: $C_{1}=C, C_{2}=0.8 C$, $C_{3}=C_{4}=0.25 C$, where $C=135$. The values of all other parameters are $a^{(d)}=30, A_{1}=1.625, a_{1}=50, B_{1}=29.333, b_{1}=66.667, A_{2}=3.250, a_{2}=100$, $B_{2}=44, b_{2}=100, r=0.56, V_{0}=6.0, e_{0}=2.5$. As inputs to the regions we used $p_{i}(t)=\left\langle p_{1}(t)\right\rangle+\tilde{p_{1}} \eta(t)$, where $\eta \sim N\left(0, s_{p_{i}}^{2}\right)$ is a normally distributed noise with the amplitudes $\tilde{p_{1}}=\tilde{p_{2}}=0.25$ and the standard deviations $s_{p_{1}}=s_{p_{2}}=1.0$. Without coupling, the inputs have the means $\left\langle p_{1}(t)\right\rangle=220,\left\langle p_{2}(t)\right\rangle=220$ and the system generated oscillations with the mean frequencies $f_{1}=9.233 \mathrm{~Hz}$ and $f_{2}=16.160 \mathrm{~Hz}$. 


\section{Simulated time series and observables of the neural mass model}

The time series generated by the system of two mutually coupled neural mass models (Eq. (26)) are presented in Fig. 8. The left panels ((a),(f), and (m)) show the corresponding coupling configurations, which are similar to those presented in Fig. 6. In the middle panels $((b),(d),(g),(i),(n)$, and $(p))$ examples of one trial of generated time series of the first $\left(y_{1}\right)$ and the second $\left(y_{2}\right)$ region are shown. The gray areas $(t \in[0.5,0.8])$ denote the time period when the couplings between regions were activated. The derivatives of the observable phases $\left(\theta_{1}\right.$ and $\left.\theta_{2}\right)$ obtained from the signals $y_{1}$ and $y_{2}$ using Hilbert transformation are shown in the right panels $((\mathrm{c}),(\mathrm{e}),(\mathrm{h}),(\mathrm{l}),(\mathrm{o}),(\mathrm{q}))$. These derivatives consist of an oscillatory activity and random fluctuations. For all regions and coupling configurations the oscillatory activities have the same frequencies as the corresponding time series. This indicates that the derivatives $d \theta_{i} / d t$ depend on the observable phases $\theta_{i}(i=1,2)$. When a region does not receive an input from the other region (Fig. 8(e) and (h))) the oscillatory activity of the derivative $d \theta_{i} / d t$ is equivalent to the forward transformation function $\rho_{i}$ times the intrinsic frequency $\omega_{i}$ (see Eq. (15) and Fig. 6). In other cases the oscillation of the derivatives are modulated by the input from the other region (Fig. 8(c),(l),(o), and (q)).

\section{E Spectral analysis of the neural mass model}

From the signals generated by the system of two mutually coupled neural mass models we obtained the observable phases by means of the Hilbert transformation. Then the derivatives of the observable phases were approximated as

$$
\dot{\theta}_{i}=\left\langle\dot{\theta}_{i}\right\rangle+\sum_{j=1, j \neq i}^{N_{r}} F_{i j}^{(n, m)} e^{\mathrm{i}\left(n \varphi_{i}+m \varphi_{j}\right)} .
$$

To find the values of $F_{i j}^{(n, m)}$, we calculated first the derivatives $\dot{\theta}_{i}$ for every time point and subtracted the mean. The resulting data points were interpolated by a surface using the nearest neighbor algorithm. Then we used the second order fast Fourier transformation to calculate the values of $F_{i j}^{(n, m)}$ for the interpolated surface. The absolute values of the coefficients are presented in Fig. 9.

The coefficients at $n=0$ are related to the Fourier coefficients of the forward transformation function $\rho(\varphi)$ (times the mean frequency). The maximum number of the Fourier terms $N_{\rho}$ for $\rho(\varphi)$ in Section 3.2 we chose as the maximal value of $|m|$ for which the coefficients $F_{i j}^{(n=0, m)}$ are significantly larger than zero. For the first region it is $|m|=1$ (Fig. 9 (a)) and is $|m|=2$ for the second region (Fig. $9(\mathrm{~b})$ ). Therefore, we set $N_{\rho}=2$.

Similarly, we found the maximum number of the Fourier terms of the coupling function $N_{q}$. Here we considered the maximal values of $|m|$ and $|n|$ for which the coefficients $\left.F_{i j}^{(n, m)}\right|_{n \neq 0, m \neq 0}$ are non zero. As one can see in Figs. 9 (a) and (b) the coefficients $F_{12 / 21}^{(-1,1)}$ and $F_{12 / 21}^{(1,-1)}$ are significantly larger than zero. Therefore, we set $N_{q}=1$ for the reconstruction of the coupling function in Section 3.2. 

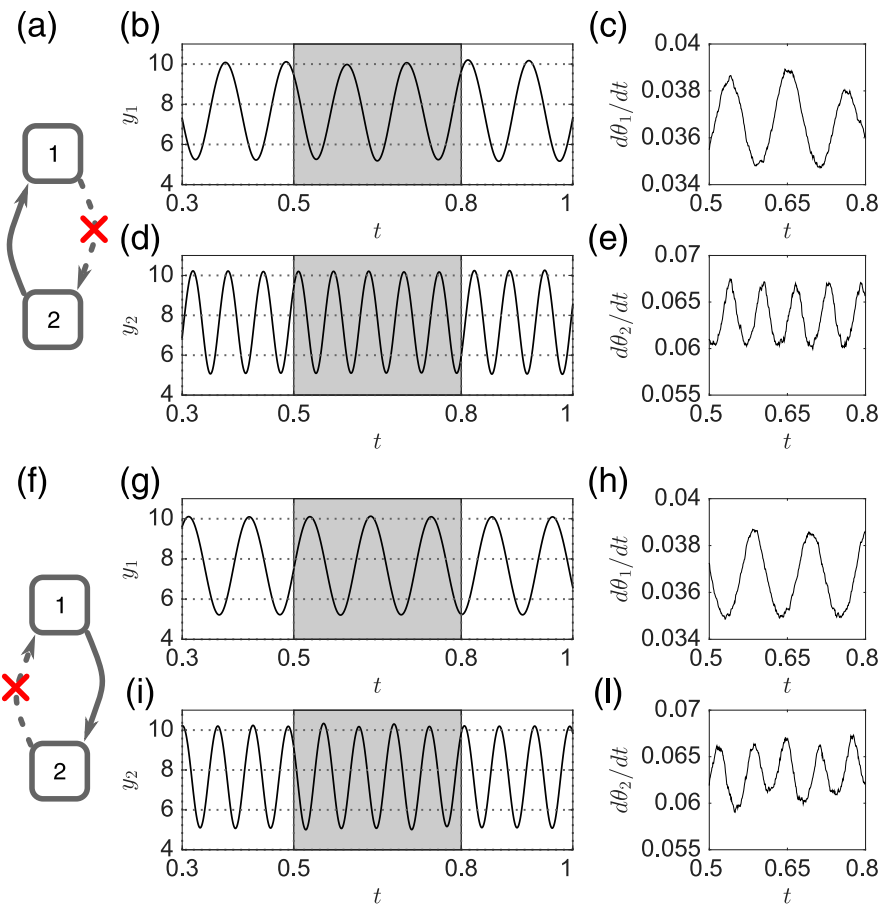

(h)

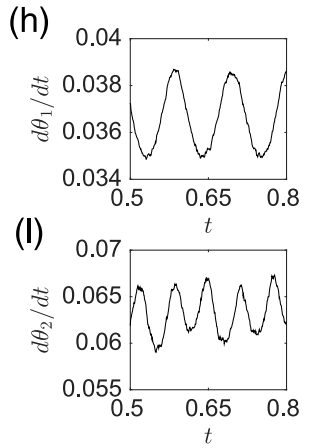

(m) (n)
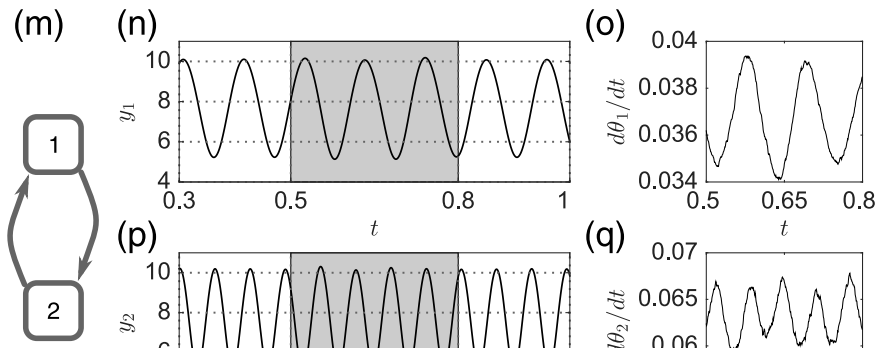

(q)

(p)

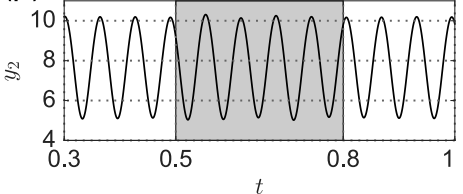

0.07

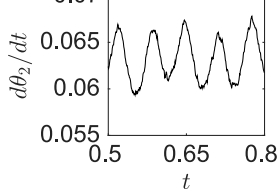

Figure 8: Example of the time series of the system of two coupled neural mass models (middle panels) for different coupling configurations (left panels) and the corresponding derivatives of the observable phases (right panels). Gray areas indicate the time interval when the couplings between regions were temporarily activated. 

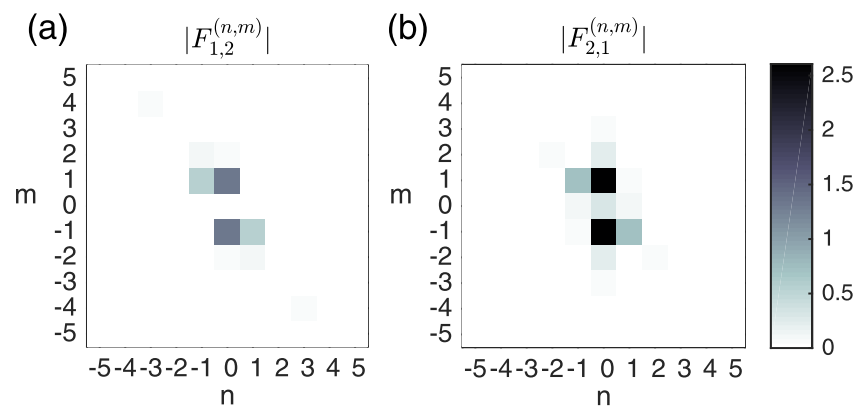

Figure 9: The norm of the Fourier coefficients of the derivatives of the observable phases obtained from the simulation of two mutually coupled neural mass oscillators (the case (c) in Fig. 6). 


\section{References}

\section{References}

Ahmadizadeh, S., Karoly, P. J., Nešić, D., Grayden, D. B., Cook, M. J., Soudry, D., Freestone, D. R., 2018. Bifurcation analysis of two coupled Jansen-Rit neural mass models. Vol. 13.

URL https://doi.org/10.1371/journal.pone.0192842

Belluscio, M. a., Mizuseki, K., Schmidt, R., Kempter, R., Buzsaki, G., 2012. Cross-Frequency Phase-Phase Coupling between Theta and Gamma Oscillations in the Hippocampus. Journal of Neuroscience 32 (2), 423-435.

URL https://doi.org/10.1523/JNEUROSCI.4122-11.2012

Buzsáki, G., 2006. Rhythms of the Brain. Oxford University Press.

Buzsáki, G., Andreas, D., Draguhn, A., Buzsaki, G., Draguhn, A., jun 2004. Neuronal Oscillations in Cortical Networks. Science 304 (June), 1926.

URL https://doi.org/10.1126/science. 1099745

Chen, C. C., Kiebel, S. J., Friston, K. J., 2008. Dynamic causal modelling of induced responses. NeuroImage 41 (4), 1293-1312.

URL https://doi.org/10.1016/j.neuroimage.2008.03.026

Chen, C. C., Kiebel, S. J., Kilner, J. M., Ward, N. S., Stephan, K. E., Wang, W. J., Friston, K. J., 2012. A dynamic causal model for evoked and induced responses. NeuroImage 59 (1), 340-348.

URL https://doi.org/10.1016/j.neuroimage.2011.07.066

Daunizeau, J., David, O., Stephan, K. E., 2011. Dynamic causal modelling: A critical review of the biophysical and statistical foundations. NeuroImage $58(2), 312-322$.

URL https://doi.org/10.1016/j.neuroimage.2009.11.062

David, O., Cosmelli, D., Friston, K. J., 2004. Evaluation of different measures of functional connectivity using a neural mass model. NeuroImage 21 (2), 659673.

URL https://doi.org/10.1016/j.neuroimage.2003.10.006

David, O., Friston, K. J., 2003. A neural mass model for MEG/EEG: Coupling and neuronal dynamics. NeuroImage 20 (3), 1743-1755.

URL https://doi.org/10.1016/j.neuroimage. 2003.07.015

David, O., Kiebel, S. J., Harrison, L. M., Mattout, J., Kilner, J. M., Friston, K. J., 2006. Dynamic causal modeling of evoked responses in EEG and MEG. NeuroImage 30 (4), 1255-1272.

URL https://doi.org/10.1016/j.neuroimage.2005.10.045

Duggento, A., Stankovski, T., McClintock, P. V. E., Stefanovska, A., 2012. Dynamical Bayesian inference of time-evolving interactions: From a pair of coupled oscillators to networks of oscillators. Physical Review E - Statistical, Nonlinear, and Soft Matter Physics 86 (6), 1-15.

URL https://doi.org/10.1103/PhysRevE.86.061126 
Friston, K., Ashburner, J., Kiebel, S., Nichols, T., Penny, W. (Eds.), 2007a. Statistical Parametric Mapping. Elsevier.

URL https://doi .org/10.1016/B978-0-12-372560-8.X5000-1

Friston, K., Henson, R., Phillips, C., Mattout, J., 2006. Bayesian estimation of evoked and induced responses. Human Brain Mapping 27 (9), 722-735.

URL https://doi .org/10.1002/hbm. 20214

Friston, K., Mattout, J., Trujillo-Barreto, N., Ashburner, J., Penny, W., 2007b. Variational free energy and the Laplace approximation. NeuroImage 34 (1), 220-234.

URL https://doi.org/10.1016/j.neuroimage.2006.08.035

Friston, K., Penny, W., Phillips, C., Kiebel, S., Hinton, G., Ashburner, J., jun 2002a. Classical and Bayesian Inference in Neuroimaging: Theory. NeuroImage 16 (2), 465-483.

URL https://doi.org/10.1006/nimg.2002.1090

Friston, K. J., 2002. Bayesian estimation of dynamical systems: an application to fMRI. NeuroImage $16(2), 513-30$.

URL https://doi.org/10.1006/nimg.2001.1044

Friston, K. J., Glaser, D. E., Henson, R. N. a., Kiebel, S., Phillips, C., Ashburner, J., 2002b. Classical and Bayesian inference in neuroimaging: applications. NeuroImage 16 (2), 484-512.

URL https://doi.org/10.1006/nimg.2002.1090

Friston, K. J., Harrison, L., Penny, W., 2003. Dynamic causal modelling. NeuroImage 19 (4), 1273-1302.

URL https://doi.org/10.1016/S1053-8119(03)00202-7

Greenblatt, R. E., Pflieger, M. E., Ossadtchi, a. E., 2012. Connectivity measures applied to human brain electrophysiological data. Journal of Neuroscience Methods 207 (1), 1-16.

URL https://doi.org/10.1016/j.jneumeth.2012.02.025

Higham., D. J., 2001. An Algorithmic Introduction to Numerical Simulation of Stochastic Differential Equations. SIAM Review 43 (3), 525-546.

URL https://doi .org/10.1137/S0036144500378302

Honeycutt, R. L., jan 1992. Stochastic Runge-Kutta algorithms. I. White noise. Physical Review A 45 (2), 600-603.

URL https://doi.org/10.1103/PhysRevA.45.600

Hoppensteadt, F. C., Izhikevich, E. M., 1997. Weakly Connected Neural Networks. Vol. 126 of Applied Mathematical Sciences. Springer New York, New York, NY.

URL https://doi.org/10.1007/978-1-4612-1828-9

Hyafil, A., Giraud, A.-l., Fontolan, L., Gutkin, B., 2015. Neural Cross-Frequency Coupling : Connecting Architectures, Mechanisms, and Functions. Trends in Neurosciences 38 (11), 725-740.

URL https://doi.org/10.1016/j.tins.2015.09.001 
Jansen, B. H., Rit, V. G., 1995. Electroencephalogram and visual evoked potential generation in a mathematical model of coupled cortical columns. Biological Cybernetics 73 (4), 357-366.

URL https://doi.org/10.1007/BF00199471

Kralemann, B., Cimponeriu, L., Rosenblum, M., Pikovsky, A., Mrowka, R., nov 2007. Uncovering interaction of coupled oscillators from data. Physical Review E 76 (5), 1-4.

URL https://doi.org/10.1103/PhysRevE.76.055201

Kralemann, B., Cimponeriu, L., Rosenblum, M., Pikovsky, A., Mrowka, R., jun 2008. Phase dynamics of coupled oscillators reconstructed from data. Physical Review E 77 (6), 1-16.

URL https://doi.org/10.1103/PhysRevE.77.066205

Kralemann, B., Pikovsky, A., Rosenblum, M., 2011. Reconstructing phase dynamics of oscillator networks. Chaos 21 (2), 1-10.

URL https://doi.org/10.1063/1.3597647

Kralemann, B., Pikovsky, A., Rosenblum, M., 2013. Detecting triplet locking by triplet synchronization indices. Physical Review E - Statistical, Nonlinear, and Soft Matter Physics 87 (5), 1-6.

URL https://doi.org/10.1103/PhysRevE.87.052904

Kralemann, B., Pikovsky, A., Rosenblum, M., aug 2014. Reconstructing effective phase connectivity of oscillator networks from observations. New Journal of Physics 16 (8), 085013.

URL https://doi.org/10.1088/1367-2630/16/8/085013

Kuramoto, Y., 1984. Chemical Oscillations, Waves, and Turbulence. Vol. 19. Springer Berlin Heidelberg, Berlin, Heidelberg.

URL https://doi.org/10.1007/978-3-642-69689-3

Lachaux, J.-p., Rodriguez, E., Martinerie, J., Varela, F. J., 1999. Measuring phase synchrony in brain signals. Human brain mapping 8, 194-208.

URL https://doi.org/10.1002/(SICI) 1097-0193(1999)8:4<194::AIDHBM4 $>3.0 . \mathrm{CO} ; 2-\mathrm{C}$

Lobier, M., Siebenhühner, F., Palva, S., Palva, J. M., 2014. Phase transfer entropy: A novel phase-based measure for directed connectivity in networks coupled by oscillatory interactions. NeuroImage $85,853-872$.

URL https://doi.org/10.1016/j .neuroimage. 2013.08.056

Lozano-Soldevilla, D., ter Huurne, N., Oostenveld, R., 2016. Neuronal Oscillations with Non-sinusoidal Morphology Produce Spurious Phase-to-Amplitude Coupling and Directionality. Frontiers in Computational Neuroscience 10 (August), Article 87.

URL https://doi.org/10.3389/fncom.2016.00087

Marreiros, A. C., Kiebel, S. J., Friston, K. J., 2008. Dynamic causal modelling for fMRI: A two-state model. NeuroImage 39 (1), 269-278.

URL https://doi.org/10.1016/j.neuroimage.2007.08.019 
Moran, R. J., Stephan, K. E., Seidenbecher, T., Pape, H. C., Dolan, R. J., Friston, K. J., 2009. Dynamic causal models of steady-state responses. NeuroImage 44 (3), 796-811.

URL https://doi.org/10.1016/j.neuroimage.2008.09.048

Penny, W. D., Litvak, V., Fuentemilla, L., Duzel, E., Friston, K., 2009. Dynamic Causal Models for phase coupling. Journal of Neuroscience Methods 183 (1), $19-30$.

URL https://doi.org/10.1016/j.jneumeth.2009.06.029

Penny, W. D., Stephan, K. E., Mechelli, A., Friston, K. J., 2004. Comparing dynamic causal models. NeuroImage 22 (3), 1157-1172.

URL https://doi.org/10.1016/j.neuroimage.2004.03.026

Pikovsky, A., Rosenblum, M., Kurths, J., 2001. Synchronization: A Universal Concept in Nonlinear Sciences. Cambridge nonlinear science series. Cambridge University Press.

Platen, E., 1987. Derivative free numerical methods for stochastic differential equations. In: Stochastic Differential Systems. Vol. 96 of Lecture Notes in Control and Information Sciences. Springer-Verlag, Berlin/Heidelberg, pp. 187-193.

URL https://doi.org/10.1007/BFb0038934

Rosenblum, M. G., Pikovsky, a. S., 2001. Detecting direction of coupling in interacting oscillators. Physical review. E, Statistical, nonlinear, and soft matter physics 64 (4 Pt 2), 045202.

URL https://doi.org/10.1103/PhysRevE.64.045202

Sauer, T., 2012. Numerical solution of stochastic differential equations in finance. Handbook of Computational Finance (1998), 529-550.

URL https://doi.org/10.1007/978-3-642-17254-0\{\_\}19

Scheffer-Teixeira, R., Tort, A. B., 2016. On cross-frequency phase-phase coupling between theta and gamma oscillations in the hippocampus. bioRxiv (m), 045963.

URL https://doi.org/10.1101/045963

Schnitzler, A., Gross, J., 2005. Normal and pathological oscillatory communication in the brain. Nature Reviews Neuroscience 6 (4), 285-296.

URL https://doi.org/10.1038/nrn1650

Spiegler, A., Kiebel, S. J., Atay, F. M., Knösche, T. R., 2010. Bifurcation analysis of neural mass models: Impact of extrinsic inputs and dendritic time constants. NeuroImage 52 (3), 1041-1058.

URL https://doi.org/10.1016/j .neuroimage.2009.12.081

Stankovski, T., Duggento, A., McClintock, P. V., Stefanovska, A., 2012. Inference of time-evolving coupled dynamical systems in the presence of noise. Physical Review Letters 109 (2), 1-5.

URL https://doi.org/10.1103/PhysRevLett.109.024101 
Stankovski, T., Pereira, T., McClintock, P. V. E., Stefanovska, A., nov 2017. Coupling functions: Universal insights into dynamical interaction mechanisms. Reviews of Modern Physics 89 (4), 045001.

URL https://doi.org/10.1103/RevModPhys.89.045001

Stankovski, T., Ticcinelli, V., McClintock, P. V. E., Stefanovska, A., 2015. Coupling functions in networks of oscillators. New Journal of Physics 17 (3), 035002.

URL https://doi.org/10.1088/1367-2630/17/3/035002

Stephan, K. E., Kasper, L., Harrison, L. M., Daunizeau, J., den Ouden, H. E. M., Breakspear, M., Friston, K. J., 2008. Nonlinear dynamic causal models for fMRI. NeuroImage 42 (2), 649-662.

URL https://doi.org/10.1016/j.neuroimage.2008.04.262

Stephan, K. E., Penny, W. D., Moran, R. J., den Ouden, H. E. M., Daunizeau, J., Friston, K. J., 2010. Ten simple rules for dynamic causal modeling. NeuroImage 49 (4), 3099-3109.

URL https://doi.org/10.1016/j.neuroimage.2009.11.015

Tak, S., Kempny, A. M., Friston, K. J., Leff, A. P., Penny, W. D., 2015. Dynamic causal modelling for functional near-infrared spectroscopy. NeuroImage 111, $338-349$

URL https://doi.org/10.1016/j.neuroimage.2015.02.035

Tort, A. B. L., Komorowski, R., Eichenbaum, H., Kopell, N., 2010. Measuring Phase-Amplitude Coupling Between Neuronal Oscillations of Different Frequencies. Journal of Neurophysiology 104 (2), 1195-1210.

URL https ://doi.org/10.1152/jn.00106.2010

van Driel, J., Cox, R., Cohen, M. X., 2015. Phase-clustering bias in phaseamplitude cross-frequency coupling and its removal. Journal of Neuroscience Methods 254, 60-72.

URL https://doi.org/10.1016/j.jneumeth.2015.07.014

van Ede, F., Quinn, A. J., Woolrich, M. W., Nobre, A. C., jul 2018. Neural Oscillations: Sustained Rhythms or Transient Burst-Events? Trends in Neurosciences 41 (7), 415-417.

URL https://doi.org/10.1016/j.tins.2018.04.004

van Wijk, B. C., Cagnan, H., Litvak, V., Kühn, A. A., Friston, K. J., 2018. Generic dynamic causal modelling: An illustrative application to Parkinson's disease. NeuroImage 181 (January), 818-830.

URL https://doi.org/10.1016/j.neuroimage.2018.08.039

Varela, F., Lachaux, J. P., Rodriguez, E., Martinerie, J., 2001. The brainweb: phase synchronization and large-scale integration. Nature reviews. Neuroscience 2 (4), 229-239.

URL https://doi.org/10.1038/35067550

Vindiola, M. M., Vettel, J. M., Gordon, S. M., Franaszczuk, P. J., McDowell, K., 2014. Applying EEG phase synchronization measures to non-linearly coupled neural mass models. Journal of Neuroscience Methods 226, 1-14.

URL https://doi.org/10.1016/j.jneumeth.2014.01.025 